\title{
Mathematical Analysis of Electrical Responses in Photoreceptor Cells Reveals Distinct Chemical Control of Visual Signal Transduction in Rods and Cones.
}

\author{
Yukari Takeda \\ University of Fukui \\ Kazuma Sato \\ Ritsumeikan University \\ Yukari Hosoki \\ Ritsumeikan University \\ Shuji Tachibanaki \\ Osaka University \\ Chieko Koike \\ Ritsumeikan University \\ Akira AMANO ( $\square$ a-amano@fc.ritsumei.ac.jp ) \\ Ritsumeikan University
}

\section{Research Article}

Keywords: Retinal photoreceptor cells, phototransduction system, photoresponses, phosphodiesterases

Posted Date: October 5th, 2021

DOl: https://doi.org/10.21203/rs.3.rs-950942/v1

License: (c) This work is licensed under a Creative Commons Attribution 4.0 International License.

Read Full License 
1 Mathematical analysis of electrical responses in photoreceptor cells reveals distinct 2 chemical control of visual signal transduction in rods and cones.

3

4 *Yukari Takeda ${ }^{1},{ }^{*}$ Kazuma Sato $^{2}$, Yukari Hosoki ${ }^{2}$, Shuji Tachibanaki ${ }^{3}$, Chieko Koike ${ }^{4}$, Akira

5 Amano $^{2}$

6

$7 \quad{ }^{1}$ Department of Integrative \& Systems Physiology, Faculty of Medical Sciences, University of

8 Fukui, Fukui City, Fukui, Japan

$9 \quad{ }^{2}$ Department of Life Sciences, Ritsumeikan University, Kusatsu City, Shiga, Japan

$10{ }^{3}$ Graduate School of Frontier Biosciences, Osaka University, Suita City, Osaka, Japan

$11{ }^{4}$ Department of Pharmaceutical Sciences, Ritsumeikan University, Kusatsu City, Shiga, Japan

13 Running header: Analysis of Light-Dependent Phototransduction in rod and cone

14 Corresponding author: Akira Amano

15 Mail address: a-amano@,fc.ritsumei.ac.jp

16

17

1-1-1 Nojihigashi, Kusatsu, Shiga, 525-8577, Japan

18 Phone number: 077-561-2584

19 Fax number: 077-561-3729

20

21

* Kazuma Sato and Yukari Takeda contributed equally to this study.

22

23

There is no commercial relationships disclosures for this research.

24 


\section{Abstract}

27 Retinal photoreceptor cells, rods and cones, convert photons of light into chemical and electrical 28 signals as the first step of the visual transduction cascade. Although the chemical processes in the 29 phototransduction system are very similar to each other in these photoreceptors, the light sensitivity 30 and time resolution of the photoresponse in rods are functionally different than those in the 31 photoresponses of cones. To systematically investigate how photoresponses are divergently regulated 32 in rods and cones, we have developed a detailed mathematical model on the basis of the Hamer model. 33 The current model successfully reconstructed light intensity-, ATP- and GTP-dependent changes in 34 concentrations of phosphorylated visual pigments (VPs), activated transducins ( $\operatorname{Tr} * \mathrm{~s}$ ) and 35 phosphodiesterases (PDEs), as well as cyclic nucleotide-gated currents $\left(I_{\mathrm{CNG}}\right)$ in rods and cones.

36 In comparison to rods, the lower light sensitivity of cones was attributed not only to the lower affinity 37 of activated VPs for Trs but also to the faster desensitization of the VPs. The assumption of an 38 intermediate inactive state, MIIi, in the thermal decay of activated VPs was pivotal for inducing faster 39 inactivation of VPs. In addition to the faster inactivation of VPs, calculating a faster rate of RGS9 40 intervention for PDE-induced Tr* inactivation in cones was indispensable for simulating the electrical 41 waveforms of the light intensity-dependent $I_{\mathrm{CNG}}$ at higher temporal resolution in experimental systems 42 in vivo. 


\section{Introduction}

Retinal photoreceptor cells, the key players in the visual system, convert photons of light into chemical and electrical signals as the first step of the visual transduction cascade. When incident light stimulates a visual pigment (VP; a prototypical G proteincoupled receptor) on membranous disks at the outer segments of photoreceptors, the catalytic activity of a heterotrimeric G-protein, transducin (Tr), of exchanging GTPs for previously bound GDP increases. The GTP-bound $\alpha$ subunit of Tr dissociates from the $\beta \gamma$ subunits and subsequently activates phosphodiesterase (PDE) [1], which in turn hydrolyzes cyclic guanosine $3^{\prime}-5^{\prime}$ monophosphate (cGMP). The signals of visual transduction are highly amplifiable since a single stimulated VP (VP*) activates $\sim 30$ and $\sim 140$ molecules of Tr per second in cones and rods, respectively [2]. Activated VPs are simultaneously inactivated through two distinct mechanisms: phosphorylation by visual pigment-specific kinases (RKs) and thermal decay processes, thereby contributing to the termination of Tr activity.

Under scotopic conditions (dark), basal cGMP regulates the activities of cyclic nucleotide-gated (CNG) nonselective cation channels, allowing a steady inward current [3]. Photoreceptor cells are thus slightly depolarized $(-40 \mathrm{mV}$, [4]) and spontaneously release a neurotransmitter, glutamate [5]. Upon stimulation of visual pigments the concentration of cGMP ([cGMP]) decreases, which deactivates the CNG channels and subsequently hyperpolarizes the photoreceptor to $\sim-60 \mathrm{mV}$, preventing the release of neurotransmitters [4]. Postsynaptic responses to glutamate may be excitatory or inhibitory depending on the postsynaptic cell type involved [6]. Signals generated by switching "on" and "off" glutamate-mediated neurotransmission are comprehensively processed within the complex network of retinal cells (bipolar, horizontal, amacrine, and ganglion cells) and sent to optic nerve fibers.

There are two classic types of photoreceptors in mammalian eyes: rods and cones [7]. Although the chemical processes through the visual transduction cascades in these photoreceptors, as described above, are very similar to each other, several functional differences have been experimentally observed. Rods are highly sensitive to light, and visual transduction in rods may be triggered by a single photon. On the other hand, cones require significantly brighter light to activate the signaling cascade and generate electrical signals [8]. Another major difference is reflected in the time course of the photoresponses. The electrical waveforms of the flash light responses are activated and deactivated relatively slowly, exhibiting a strikingly prolonged response in rods than is observed in cones [8]. The differences in sensitivity and the temporal resolution of the light responses in rods and cones are attributed to different reaction rates throughout 
the visual phototransduction system. However, the quantitative aspects of the molecular mechanisms underlying distinct phototransduction systems have not yet been conclusively clarified [9]. To quantitatively and systematically investigate how light intensity-dependent photoresponses are divergently regulated in rods and cones, a systems analysis of visual signal transduction systems for these photoreceptors is indispensable.

Former mathematical models of visual transduction cascades for rods and cones, the Hamer [10] and Dell'Orco [11] models, explain fundamental molecular reactions in the phototransduction system. Hamer et al. [10] and Dell'Orco et al. [11], however, seemed to place particular emphasis on photoresponses, especially single photon responses (SPRs). Their models thus generate macroscopic electrical events, including SPRs, but do not faithfully reproduce some of the microscopic biochemical responses, e.g., changes in concentrations and activity levels of $\mathrm{VP}^{*}$, Tr, or PDE, although these biochemical parameters were described thoroughly in the literature [2] [8] [1]. Furthermore, these previously developed mathematical models do not take into account [ATP] or [GTP]; therefore, ATP-dependent phosphorylation and the subsequent desensitization of VP* and GTP-dependent activation of Tr and cGMP production are not accurately estimated. These models remain powerful for demonstrating scientific aspects of the phototransduction system underlying photoresponses, especially SPRs; however, these models are not sufficient for practical application, e.g., in electroretinogram (ERG) simulation, where specific predictions of the molecular defects in microscopic biochemical reactions underlying pathophysiological electrical responses ( [12] [13]) are required in medicine. In the present study, frameworks for all the molecular reactions of phototransduction cascades were mathematically elaborated on the basis of the Hamer model [10] to ensure that a wide variety of light intensity-, ATP-, and GTP-dependent microscopic biochemical reactions underlying macroscopic electrical responses are accurately reconstructed.

The proposed lower vertebrate rod and cone models successfully reproduced in vitro time courses of light intensity-, ATP- and GTP-dependent changes in the concentrations of phosphorylated VPs, $\operatorname{Tr}^{*} \mathrm{~s}$ and PDEs and of cyclic nucleotide-gated currents $\left(I_{\mathrm{CNG}}\right)$ in rods and cones from frog and carp [14] [15]. Compared to that of rods, the lower light sensitivity of cones was attributed to the lower affinity of the activated VPs for Trs, as well as the faster desensitization (phosphorylation and inactivation) of VPs. The assumption of an intermediate inactive state, MIIi, during the thermal decay of activated VP was pivotal for inducing faster inactivation of VP. Furthermore, the combination of faster rates of VP desensitization and RGS9-mediated Tr* inactivation together were indispensable for simulating higher 
temporal resolution of the electrical waveforms of the light intensity-dependent $I_{\mathrm{CNG}}$ in vivo experimental systems.

\section{Methods}

A dynamic mathematical model of the visual transduction system in rods and cones was constructed to analyze two distinct sets of experimental observations: fundamental microscopic "in vitro" biochemical reactions in phototransduction (Figures 2-4) and light intensity-dependent macroscopic photoresponses observed in "intact" retinal photoreceptor cells (Figure 5).

The fundamental component of the proposed "in vitro" model was developed on the basis of the Hamer model [10] and Dell'Orco model [11] by elaborating the frameworks for all the key molecular reactions of phototransduction cascades that account for the details of the underlying biochemistry as described in the subsections below. The molecular environment of the membranous disk where all chemical reactions for phototransduction take place in vivo (e.g., concentrations and spatial distributions of membrane-associated molecules, VP, RK, Tr, and PDE, present at the outer segments of photoreceptors), was assumed to be preserved and thus nearly identical to that isolated for biochemical experiments (see Figures 2-4). Taking into account the molecular environment of a single membranous disk, the in vivo concentrations of VP, RK, PDE, and were determined (Wet column in Table 1, see the Discussion section for more details).

136 Note that different concentrations of freely diffusing factors, ATP, GTP, and cGMP, were applied depending on the corresponding biochemical experiments. The simulated results in Figures 2-4 were all reproduced using the parameters listed in Table 1 "Sim." column and Table 3 , in the "in vitro" model columns. The results are expressed as events per either VP* or VPtot.

For the calculations of the light intensity-dependent macroscopic photoresponses

143 e.g., arrestin (Arr) and G protein signaling 9 (RGS9), were considered in addition to the

144 "in vitro" system. The ICNG presented in Figure 5 were calculated using the parameters

145 listed in Table 3, in the "intact" model columns, and as described in detail in each 146 subsection below.

147 The reaction scheme of the visual transduction cascade is shown in Figure 1, and 148 the differential equations for the reaction steps in the model are described in the 149 Supplementary materials under Equations. The abbreviations of signaling molecules are 150 summarized in Table 2. The parameters used to define the present model, including the 
concentrations and binding constants $\left(K_{d}\right)$ of signaling factors and the rate constant $(k)$, maximum activity $\left(V_{\max }\right)$ and half-maximal effective concentration $\left(K_{1 / 2}\right)$ of substances for activation of enzymes, among other parameters, are listed in Table 3. The initial values of some variables are also listed in Table 4. Model development and simulation-based analyses were both performed with simBio [16]. The time integration of the differential equations was conducted using the Euler method with a time step of $1 \mu \mathrm{s}$.

A wide variety of light intensity-, ATP-, and GTP-dependent photoresponses in distinct types of photoreceptors in frogs and carp (extensively described by the biochemical experiments of Kawamura et al. [14] [2], see Figures 2-5) were successfully reconstructed and then quantitatively and systematically investigated in the current study.

\section{Activation of visual pigments}

Light stimulation activates a VP, which consists of an opsin combined with the chromophore 11-cis-retinal. The activation of a VP is initiated by light-induced isomerization of 11-cis-retinal to the all-trans form, causing conformational changes in the opsin. The intensity of the light stimulus $\left(I_{\text {light }}\right)$, indicated in Figures 2-4, are given as a percentage (\%), reflecting the \% of visual pigments activated (bleached) by the light stimulus when considering the concentration of the total visual pigment as $100 \%$ (1).

$I_{\text {light }}=\frac{\left[V P^{*}\right]}{\left[V P_{\text {total }}\right]}$

Activated VP immediately after light stimulation $\left(\mathrm{MII}_{0}^{*}\right.$ at $\left.\mathrm{t}=0\right)$ is thus determined by (2).

$$
\left[M I I_{0}^{*}\right]=I_{\text {light }}\left[V P_{\text {total }}\right]
$$

In the current simulation study, the stimulated VP, $\mathrm{MII}^{*}\left(\mathrm{MII}_{0}{ }_{0}-\mathrm{MII}^{*}{ }_{3}\right.$, see Figure 1$)$, is assumed to be capable of activating Tr, while being simultaneously inactivated through two distinct mechanisms: phosphorylation and thermal decay.

\section{Phosphorylation of visual pigments}

The phosphorylation of VPs is mediated by visual pigment-specific kinases (RKs). 
186 In the Hamer model, phosphorylation reactions were simply calculated in 3 reaction 187 steps: binding of the activated VP to an RK, phosphorylation of VP, and the dissociation 188 of the RK from the VP. The Hamer model cannot be used to simulate the [ATP]189 dependent phosphorylation or sequential inactivation of the VPs. Therefore, the binding 190 of ATP to and dissociation of ADP from the MII*-RK complex were incorporated into the 191 current model (see the reaction scheme in Figure 1B, reaction formulae R.1.1 - 1.8 below 192 and the corresponding equations, Eqs. S1 - 26, S51 - 60, in the Supplementary materials 193 under Equations) to ensure that the activities of Tr and PDE in the presence and absence 194 of ATP, as estimated by experimental studies, are reconstructed and considered in this 195 study (Figures 3 and 4)

196

$$
M I I_{n}^{*}+R K_{\text {free }} \underset{k_{R K 2}}{\stackrel{k_{R K 1}}{\longrightarrow}} M I I_{n}^{*} \cdot R K_{\text {pre }}
$$

$$
M I I_{n}^{*} \bullet R K_{\text {pre }}+A T P \underset{k_{R K 4}}{\stackrel{k_{R K 3}}{\rightleftarrows}} M I I_{n}^{*} \cdot R K_{\text {pre }} \cdot A T P
$$

$$
M I I_{n}^{*} \cdot R K_{\text {pre }} \cdot A T P \stackrel{k_{R K 5}}{\longrightarrow} M I I_{n+1}^{*} \cdot R K_{\text {post }}+A D P
$$

$$
M I I_{n+1}^{*} \cdot R K_{\text {post }} \stackrel{k_{R K 6}}{\longrightarrow} M I I_{n+1}^{*}+R K_{\text {free }}
$$

$$
M I I_{n}^{i}+R K_{\text {free }} \underset{k_{R K 2}^{i}}{\stackrel{k_{R K 1}^{i}}{\rightleftarrows}} M I I_{n}^{i} \bullet R K_{\text {pre }}
$$

$$
M I I_{n}^{i} \cdot R K_{\text {pre }}+A T P \underset{k_{R K 4}^{i}}{\stackrel{k_{R K 3}^{i}}{\longrightarrow}} M I I_{n}^{i} \cdot R K_{\text {pre }} \cdot A T P
$$

$$
M I I_{n}^{i} \cdot R K_{p r e} \cdot A T P \stackrel{k_{R K 5}^{i}}{\longrightarrow} M I I_{n+1}^{i} \cdot R K_{\text {post }}+A D P
$$

$$
M I I_{n+1}^{i} \cdot R K_{\text {post }} \stackrel{k_{R K 6}^{i}}{\longrightarrow} M I I_{n+1}^{i}+R K_{\text {free }}
$$

where $0 \leq \mathrm{n} \leq 3$ ( $\mathrm{n}=$ number of phosphorylated VP sites) when simulating photoresponses in vitro (Figures $2-4$ ) and $0 \leq \mathrm{n} \leq 1$ when simulating $I_{\text {photo }}$ recorded under in vivo conditions (Figure 5). The notation "pre" and "post" in above reaction distinguish RK-bound states of $\mathrm{R}^{*}$ before and after phosphorylation, respectively. $\sim 3$, which is in good agreement with the number of phosphates necessary for the 
complete suppression of the activated VPs in rods [18]. Although a maximum of 9

213 phosphorylation sites in the C terminus of MII have been suggested [19], only 3 sites

214 were considered in the current model (for Figures 2-4, $0 \leq \mathrm{n} \leq 2$ for R.1.1 - 1.8) based

215 on biochemical experiment observations in vitro [18] [17]. For the calculation of the

216 visual pigment phosphorylation shown in Figure 2, the total number of phosphate groups

217 incorporated into activated visual pigments was determined by Eq. (3).

218

$$
\begin{aligned}
{[\mathrm{Pi}] } & =\sum_{\mathrm{n}=1}^{2}\left\{\left(\left[\mathrm{MII}_{\mathrm{n}}^{*} \bullet \mathrm{RK}_{\mathrm{pre}}\right]+\left[\mathrm{MII}_{\mathrm{n}}^{*} \bullet \mathrm{RK}_{\mathrm{pre}} \bullet \mathrm{ATP}\right]+\left[\mathrm{MII}_{\mathrm{n}}^{\mathrm{i}} \bullet \mathrm{RK}_{\mathrm{pre}}\right]\right.\right. \\
& \left.\left.+\left[\mathrm{MII}_{\mathrm{n}}^{\mathrm{i}} \bullet \mathrm{RK}_{\mathrm{pre}} \cdot \mathrm{ATP}\right]\right) \times \mathrm{n}\right\}+\sum_{\mathrm{n}=1}^{3}\left\{\left(\left[\mathrm{MII}_{\mathrm{n}}^{*}\right]+\left[\mathrm{MII}_{\mathrm{n}}^{*} \bullet \mathrm{RK}_{\mathrm{post}}\right]\right.\right. \\
& \left.\left.+\left[\mathrm{MII}_{\mathrm{n}}^{\mathrm{i}}\right]+\left[\mathrm{MII}_{\mathrm{n}}^{\mathrm{i}} \bullet \mathrm{RK}_{\text {post }}\right]\right) \times \mathrm{n}\right\}
\end{aligned}
$$

Rate constants for the phosphorylation reactions of the VPs, $k_{\mathrm{RK} 1}-k_{\mathrm{RK} 6}$, were estimated by model fitting to the experimental data exhibiting the time courses and the maximal rate of phosphorylation in rod and cone at given light flash intensities [17] (see Figure 2) The reaction rates for the phosphorylation of the activated VPs in the Hamer model were designed to vary as the phosphorylation reactions proceed, whereas those for the current model were set as constant parameters since the rate of phosphorylation up to $3 \mathrm{P}_{\mathrm{i}} / \mathrm{VP}^{*}$ total does not seem to vary significantly (see Fig. 1 in Tachibanaki et al., [17]). The phosphorylation rate constants for $\mathrm{MII}^{\mathrm{i}}, k^{\mathrm{i}} \mathrm{RK} 1-k^{\mathrm{i} K 6}$, were assumed to be one-half those of $\mathrm{MII}^{*}\left(k_{\mathrm{RK} 1}-k_{\mathrm{RK} 6}\right)$ to reproduce continuing phosphorylation processes even after VP activity was completely terminated (based on the comparison of the time course of VP phosphorylation (see Figure $2 A$ ) with that of Tr activation in the presence of ATP (Figure 3A)) [17] [20].

For the simulation of the electrical waveforms of the light intensity-dependent photoresponses shown in Figure $5 B$, the phosphorylation reaction was calculated only for a single site ( $n=0$ for R.1.1 - 1.8), assuming a complete loss of VP activity quickly after arrestin (Arr) binds to MII* in the "intact" system. In this case, MII*0 was assumed to be only capable of activating $\operatorname{Tr}$ (see the Discussion section).

\section{Thermal decay of visual pigments}

Thermal decay of VPs is another pathway for the inactivation mechanism. The process is mediated by conformational changes in which $\mathrm{MII}_{0}^{*_{0}}$ transitions to MIII [21] directly or after undergoing a transition to an intermediate state, MII ${ }^{\mathrm{i}}$ [22] (see the Discussion section), as well as by bleaching (dissociation of retinal from opsin) and the subsequent 
recycling of VPs [23]. MIII may also undergo bleaching processes (see the reaction

246 scheme in Figure 3, reaction formulae R.2.1 - 2.6 below, and corresponding equations,

247 Eqs. S1 - 4, S14 - 17, S27 - 28, S61 - 62, in the Supplementary materials under Equations).

Rate constants for the inactivation reaction of the VPs, $k_{2}-k_{4}$, were determined based on the reports of Kolesnikov et al. [24] and Kawamura et al. [2], while $k_{1}$ was estimated by model fitting. Note that the rates of $k_{2}-k_{4}$ were assumed to be 6 -fold faster, while $k_{1}$ was 120 -fold faster in cones than in rods, since the lifetime of the MII intermediate was expected to be exceedingly shorter in cones. The reaction rates for inactivation were verified by reproducing Tr activation in the absence of ATP under the condition that no VP phosphorylation occurs, as shown in Figure 3.

\section{Activation and inactivation of transducins}

Transducin (Tr) is a prototypic heterotrimeric $G$ protein comprising $G_{\alpha}$ and $G_{\beta \gamma}$ subunits. Stimulated visual pigments increase the catalytic activity of Tr by exchanging GTP for the GDP previously bound to $\mathrm{G}_{\alpha}$. $\mathrm{G}_{\alpha}$-GTP then dissociates from the $\mathrm{G}_{\beta \gamma}$ subunits and subsequently binds to a PDE, increasing the degradation rate of cGMP (see the reaction scheme in Figure 3, reaction formulae R.3.1 - 3.5 below, and corresponding equations, Eqs. S29 - 42, S63 - 67, in the Supplementary materials under Equations). In the experiment, the number of GTP $\gamma \mathrm{S}$, a nonhydrolyzable GTP analog, incorporated per $\operatorname{Tr} *\left(\mathrm{G}_{\alpha}-\mathrm{GTP} \gamma \mathrm{S}\right)$ was measured as an indicator of activated $\operatorname{Tr}$.

$$
\begin{array}{ll}
275 & M I I_{n}^{*}+G \cdot G D P \stackrel{k_{G 1 n}}{\underset{k_{G 2}}{\longrightarrow}} M I I_{n}^{*} \bullet G \cdot G D P \\
276 & M I I_{n}^{*} \bullet G \cdot G D P \stackrel{k_{G 3} \underset{k_{G 4}}{\longrightarrow}}{\leftrightarrows} M I I_{n}^{*} \bullet G+G D P \\
277 & M I I_{n}^{*} \bullet G+G T P \stackrel{k_{G 5}}{\longrightarrow} M I I_{n}^{*} \bullet G \cdot G T P \\
278 & M I I_{n}^{*} \bullet G \cdot G T P \stackrel{k_{G 6}}{\longrightarrow} M I I_{n}^{*}+G \cdot G T P
\end{array}
$$


where $n$ indicates the number of phosphorylated sites $(n \geq 0)$.

For the current model, the constraint of mass conservation was newly introduced to the concentration of $\operatorname{Tr}$ to prevent the continuous increase in $\mathrm{G}_{\alpha}-\mathrm{GTP} \gamma \mathrm{S}$ in response to a stronger light stimulus (see Figure $3 B$ ). Since GTP $\gamma \mathrm{S}$ is not hydrolyzable, the initial rate of the change in $\mathrm{G}_{\alpha}-\mathrm{GTP} \gamma \mathrm{S}$ (Figure $3 A$ ) purely reflects the rate of $\mathrm{Tr}$ activation because the VP* desensitization (inactivation and phosphorylation) reactions progress more slowly than those of $\operatorname{Tr}$ activation [25]. The rate constants for the reactions of $\operatorname{Tr}$ activation, $k_{\mathrm{G} 1, \mathrm{n}}-k_{\mathrm{G} 7}$, were thus estimated by model fitting to the initial rate of $\mathrm{Tr}$ activation in response to light flash stimulation ( $0.0085 \%$ for rods and $0.25 \%$ for cones) as well as the light intensity-dependent activation of $\mathrm{Tr}$ in vitro [25] [20] (see Figure 3). Note that the affinity of $\mathrm{G}_{\alpha}$-GTP for $\mathrm{MII}_{0}^{*}\left(k_{\mathrm{G} 1,0} / k_{\mathrm{G} 2}\right)$ was approximately 6 -fold higher in rods than in cones. The estimation was comparable to that of Chen et al. [26].

The $\mathrm{G}_{\alpha}$-GTP-binding rate to $\mathrm{MII}^{*}{ }_{1}\left(k_{\mathrm{G} 1,1}\right)$ was estimated to be $\sim 60 \%$ of that to $\mathrm{MII}_{0}{ }_{0}\left(k_{\mathrm{G} 1,0}\right)$, while the rate ( $k_{\mathrm{G} 1,2}$ and $k_{\mathrm{G} 1,3}$, see R.3.6) was assumed to further decrease through successive phosphorylation of the VPs (to $\mathrm{MII}^{*}{ }_{2}$ and $\mathrm{MII}^{*}{ }_{3}$, respectively), based on Gibson et al. [27] (see the Discussion section for more details).

299

reaction rates for VP inactivation were verified by reproducing the experimental data obtained in the absence of ATP (Figure 3) under the condition that VP is not phosphorylated. The values of $k_{\mathrm{G} 1, \mathrm{n}}-k_{\mathrm{G} 7}$ determined in the present study were comparable to these of the Hamer model [10]. bound GTP to GDP on a minute time scale [20] (see the reaction scheme in Figure 1, reaction formulae R.4.1 - 4.3 below, and corresponding equations, Eqs. S42 - 45, S67 - 69, in the Supplementary materials under Equations). Calculating the elution of $\mathrm{Tr}^{*}$ from the localized outer membrane complex where phototransduction takes place in experimental systems in vitro was indispensable for the reconstruction of the decay of PDE activity (Figure 4). Notably, inactivation does not occur with GTP $\gamma$ S. 
The GTPase activity of PDE-associated $\mathrm{G}_{\alpha}$ dramatically increases as the PDE $\cdot \mathrm{G}_{\alpha} \mathrm{GTP}$ complex binds to Regulator of RGS9, a GTPase-accelerating protein (see the reaction formulae R.7.1 - 7.2 below and corresponding equations, Eqs. S69 and 71, in the Supplementary materials under Equations). The RGS9 effects on free $\operatorname{Tr}^{*}\left(G_{\alpha}-G T P\right)$ were assumed to be negligible since the binding affinity of RGS9 for free $\operatorname{Tr}^{*}$ was considerably lower than it was for PDE-bound $\operatorname{Tr}^{*}$ [28]

$R G S 9 \cdot P D E^{*} \cdot G_{\alpha} \cdot G T P \stackrel{k_{R G S 3}}{\longrightarrow} P D E+G_{\alpha} \cdot G D P+P i+R G S 9$

The rate constants for RGS9-dependent hydrolysis, $k_{\mathrm{RGS} 1}-k_{\mathrm{RGS}}$, were estimated by model fitting to the time courses of the electrical waveforms of the light intensitydependent photoresponses, which are shown in Figure 5 recorded in a physiological condition [8] (see the Discussion section). Based on Tachibanaki et al. [20], an approximate 20-fold higher expression of RGS9 in cones than in rods was assumed for the simulation (see Table 3). The effect of RGS9 was excluded from the current model for the simulation of the in vitro experiments (Figures 2-4) under the assumption that RGS9 is translocated from the membranous disk in the outer segments to the inner segments of photoreceptors during sample preparation [29] (see the Discussion for more details).

\section{Regulation of PDE activity}

The catalytic activity of PDE in hydrolyzing cGMP at rest is elevated when the inhibitory $\gamma$ subunit is removed from the enzyme upon binding activated $\operatorname{Tr}\left(\mathrm{PDE}^{*} \cdot \mathrm{G}_{\alpha^{-}}\right.$ GTP, see R.5.1 - 6.2). The activity of PDE thus decreases as $\mathrm{G}_{\alpha}$-GTP is hydrolyzed by its GTPase activity. For the Hamer model, cGMP hydrolysis by PDE was simply described by a rate constant [10]. In contrast, in the current study, the chemical reaction of cGMP binding to PDE was calculated to indicate that cGMP hydrolysis (R.5.4) is also a [cGMP] $\mathrm{i}^{-}$ dependent process (see the reaction scheme in Figure 1, reaction formulae R.5.1 - 6.4 below, and corresponding equations, Eqs. S42, S44-S45 and S67-S70, in the Supplementary materials under Equations). Notably, the PDE-independent basal degradation of cGMP was also assumed when the calculating steady-state $[c G M P]_{i}$ in the dark, taking into account the continuous cGMP generation by guanylate cyclase (GC) in vivo (see Figure 5). 
Based on Kawamura et al. [8], $\mathrm{G}_{\alpha}$-GTP was also assumed to be eluted from the localized outer membrane complex where phototransduction takes place in the in vitro system (see R.6.1 and the Discussion section). The eluted $\mathrm{G}_{\alpha}$-GTP was also presumed to be inactivated, as described in the previous section (see R.6.2). The elution of $\mathrm{G}_{\alpha}$-GTP was estimated to be negligible in the "intact" system when simulating the electrical waveforms of the light intensity-dependent photoresponses in Figure 5.

362

$$
\begin{aligned}
& G_{\alpha} \cdot G T P \stackrel{k_{\text {elution }}}{\longrightarrow} G_{\alpha} \cdot G T P_{\text {eluted }} \\
& G_{\alpha} \cdot G T P_{\text {eluted }}^{\stackrel{k_{\text {decay }}}{\longrightarrow}} G_{\alpha} \cdot G D P+P i
\end{aligned}
$$

The rate constants for the reactions of PDE activation ( $k_{\mathrm{P} 1}$ and $k_{\mathrm{P} 2}$ ), cGMP hydrolysis $\left(k_{\mathrm{P} 3}, k_{\mathrm{P}} 4\right.$, and $\left.\beta_{\text {sub }}\right)$ and $\operatorname{Tr}^{*}\left(\mathrm{G}_{\alpha}-\mathrm{GTP}\right)$ inactivation $\left(k_{\text {decay }}\right)$ were estimated by model fitting to the time courses of cGMP hydrolysis per $\mathrm{VP}^{*}$ in response to a given light flash stimulation and to light intensity-dependent PDE activity [2] [8] [30] (see Figure 4). between the rate of cGMP production and degradation. The rate of GTP-dependent 
383 The half-maximal value of the cytosolic $\mathrm{Ca}^{2+}$ concentration $\left(\left[\mathrm{Ca}^{2+}\right]_{\mathrm{i}}\right)$ for the inhibition of $384 \mathrm{GC}(\mathrm{Kc})$ and the Hill coefficient for $\mathrm{Ca}^{2+-}$ dependent inhibition were determined based on

385

386

387

388

389

390

391

392

393

394

395

396

397

398

399

400

401

402

403

404

405

406

407

408

409

410

411

412

413

414

415

416

417 experimental reports [31] [32] [33] [34]. The rate constants for cGMP production, $k_{\mathrm{GC} 1} \sim$ $k_{\mathrm{GC} 3}$, and for basal cGMP degradation, $\beta_{\text {dark, }}$ were estimated by adjusting the resting cGMP level at $2 \mu \mathrm{M} \quad$ [35] given that $\left[\mathrm{Ca}^{2+}\right]_{\mathrm{i}}$ in the dark is $\sim 500 \mu \mathrm{M}$ [36] [37] [38]. The values of $k_{\mathrm{GC} 1}-k_{\mathrm{GC} 3}$ and $\beta_{\text {dark }}$ determined in the present study were comparable to those estimated by Kawamura et al. [8] and other experimental studies [39] [10] [40] [41] [15].

\section{Regulation of CNG currents in the "intact" system}

[cGMP] $]_{i}$ regulates $\mathrm{CNG}$ currents $\left(I_{\mathrm{CNG}}\right)$ in the outer segments of photoreceptor cells (see the corresponding differential equation, Eq. S83, in the Appendix under Equations). The half-maximal value of $[\mathrm{cGMP}]_{\mathrm{i}}$ for the activation of $I_{\mathrm{CNG}}\left(K_{\mathrm{m}}\right)$ and the Hill coefficient for its [cGMP] $]_{i}$-dependent activity $\left(\mathrm{n}_{\mathrm{h}}\right)$ was determined based on experimental reports [42] [43] [44] [45]. The maximum conductance of $I_{\mathrm{CNG}}, G_{\mathrm{max}}$, in the dark was estimated by reproducing the steady-state current of $\sim 4$ and $\sim 12 \mathrm{pA}$ in the rods and cones, respectively, given that membrane potential $\left(\mathrm{V}_{\mathrm{m}}\right)$ of each photoreceptor cells under the scotopic conditions is $\sim 40 \mathrm{mV}$ [46] [47].

\section{Regulation of $\left[\mathrm{Ca}^{2+}\right]_{i}$ in the "intact" system}

$\left[\mathrm{Ca}^{2+}\right]_{\mathrm{i}}$ in the current model is regulated by $\mathrm{Ca}^{2+}$ influx through $I_{\mathrm{CNG}}, \mathrm{Ca}^{2+}$ efflux via $\mathrm{Na}^{+} / \mathrm{Ca}^{2+-} \mathrm{K}^{+}$exchangers (NCKX), and $\mathrm{Ca}^{2+}$ binding to endogenous calcium buffer (see the reaction scheme in Figure $1 C$ and the corresponding differential equations in the Appendix under Equations: for free $\mathrm{Ca}^{2+}\left(\left[\mathrm{Ca}^{2+}\right]_{\mathrm{i}}\right)$, Eq. S81, and for bound $\mathrm{Ca}^{2+}(\mathrm{Cab}), \mathrm{Eq}$. S82).

The relative $\mathrm{Ca}^{2+}$ permeability of $I_{\mathrm{CNG}}(b)$ was set at 0.3 and 0.41 for rods and cones, respectively [48]. The minimum $\mathrm{Ca}^{2+}$ concentration within cells $\left(c_{0}\right)$ was set at $0.01 \mu \mathrm{M}$ based on experimental reports [10] [36] [38]. The conductance of the NCKX (YCa), total content of endogenous $\mathrm{Ca}^{2+}$ buffer $(e \mathrm{~T})$ and the rate constants for $\mathrm{Ca}^{2+}$ binding to the endogenous buffer $\left(k_{\mathrm{b} 1}\right.$ and $\left.k_{\mathrm{b} 2}\right)$ in rods and cones were estimated by reproducing the resting $\left[\mathrm{Ca}^{2+}\right]_{\mathrm{i}}$ at $500 \mathrm{nM}$ for both types of photoreceptor cells under scotopic conditions, considering that $\left[\mathrm{Ca}^{2+}\right]_{\mathrm{i}}$ may be reduced to $\sim 100 \mathrm{nM}$ when $I_{\mathrm{CNG}}$ decrease to $0 \mathrm{pA}$ in response to a strong light stimulus. The values of $\mathrm{YCa}_{\mathrm{Ca}}$ determined in the present study were comparable to those estimated by Hamer et al. [40]. 
420 Results

Phosphorylation of visual pigments

Activated VPs undergo processes of inactivation mediated by visual pigment-specific kinases (RKs). Time courses of visual pigment phosphorylation in membrane preparations of rods and cones in response to given light flash stimulation (Figure $2 \mathrm{~A}$, $1.3 \%$ for rods (a, circle) and $2.5 \%$ for cones ( $b$, triangle)) were reported by Tachibanaki et al. [17]. VP phosphorylation in cones was evidently faster than it was in rods (halfmaximum phosphorylation: $\sim 12.5 \mathrm{sec}$ in rods and $\sim 250 \mathrm{~ms}$ in cones), whereas the rate of phosphorylation depended on the flash intensity (see below). The in vitro elements of the current visual transduction cascade model well reproduced the time course of phosphorylation of visual pigments in both rods and cones (see dotted lines). The simulation study clarified that the difference in the apparent rates of phosphorylation in these two types of photoreceptors was due to distinct amounts of receptor kinases (12 $\mu \mathrm{M}$ in rods and $120 \mu \mathrm{M}$ cones) and reaction rates for each chemical process during the phosphorylation of the VPs in the rods and cones $\left(k_{\mathrm{RK} 1}-k_{\mathrm{RK} 6}\right.$ and $k^{\mathrm{i}} \mathrm{RK} 1-k_{\mathrm{RK}}$, see Table 3), as predicted by Tachibanaki et al. [17].

438 Tachibanaki and colleagues have also reported distinct maximum rates of 439 phosphorylation at different flash intensities (Figure $2 B$ ) in membrane preparations of 440 rods ( $a$, circle) and cones ( $b$, circle) [17]. Fitting the experimental results by the 441 Michaelis-Menten equation $\left(V S=V_{\max } /\left(S+K_{m}\right)\right.$, where $\mathrm{S}=\mathrm{VP}^{*} / \mathrm{VP}_{\text {tot }}$ (expressed as a 442 percentage)) yielded an estimate of $V_{\max }$ (the maximum phosphorylation rates) and $K_{\mathrm{m}}$ 443 (the half-maximal values of $\mathrm{VP}^{*} / \mathrm{VP}_{\text {tot }}$ upon stimulation of the $\mathrm{RKs}$ ) for the 444 phosphorylation reactions of $0.0049 \mathrm{Pi} / \mathrm{VP}^{*} / \mathrm{s}$ (or $0.037 \mathrm{pmol} \mathrm{Pi} / \mathrm{s}$ ) and $0.61 \%$ in rods and $4450.41 \mathrm{Pi} / \mathrm{VP}^{*} / \mathrm{s}$ (or $3.1 \mathrm{pmol} \mathrm{Pi} / \mathrm{s}$ ) and $10 \%$ in cones, respectively. $V_{\max }$ and $K_{\mathrm{m}}$ were 446 determined by fitting the same equation to the corresponding simulation results for rods 447 (Figure 2Ba, triangle) and cones (Figure 2Bb, triangle), and comparable values were 448 obtained $\left(0.0018 \mathrm{Pi} / \mathrm{VP}^{*} / \mathrm{s}\right.$ and $0.36 \%$ in rods; $0.32 \mathrm{Pi} / \mathrm{VP}^{*} / \mathrm{s}$ and $4.8 \%$ in cones, 449 respectively), indicating that the current model well reconstructed the flash intensity450 dependent phosphorylation of VPs in rods and cones. These results showed that, with 451 the chosen rate constants for the phosphorylation reaction of the VPs $\left(k_{\mathrm{RK} 1}-k_{\mathrm{RK} 6}\right.$ and $\left.452 k^{\mathrm{i} R K 1}-k^{\mathrm{i} R K 6}\right)$ in both photoreceptors, the model provided reasonable descriptions of the 453 experimental data. 

Stimulated visual pigment increases the catalytic activity of Tr in exchanging GTP for GDP. The time courses of Tr activation in the membrane preparations of rods and cones in response to given light stimulation (Figure $3 A, 0.0085 \%$ for rods (a, circle) and $0.25 \%$ for cones ( $b$, triangle)), in the presence (filled symbols) and absence of ATP (open symbols) were reported by Tachibanaki et al. [20]. For these experiments, the number of GTP $\gamma \mathrm{S}$ molecules, a nonhydrolyzable GTP analog, incorporated per VP* was measured as an indicator of activated Tr. The time course of GTP $\gamma \mathrm{S}$ binding to $\mathrm{Tr}$ in the absence of ATP, thus without VP phosphorylation, was fitted with a simple exponential function $(Y=$ $A[1-\exp (-k t)])$. The estimated initial rates $(A \mathrm{k})$ of $\operatorname{Tr}$ activation were 143 and 30 $\operatorname{Tr} * / V P * / s$ for rods and cones, respectively, suggesting that amplification of the incoming light signal at the level of Tr activation was approximately 5 -fold more efficient in rods than it was in cones. Even in the absence of ATP, GTP $\gamma \mathrm{S}$-binding reactions (A) led to eventual saturation ( 497 GTP $\gamma \mathrm{S} / \mathrm{VP}^{*}$ in rods and $\sim 8.5 \mathrm{GTP} \gamma \mathrm{S} / \mathrm{VP} *$ in cones) due to termination of $\mathrm{MII}_{0}{ }_{0}$ activity because of inactivation processes. In the presence of ATP ( $1 \mathrm{mM}$ ), the maximum amount of GTP $\gamma \mathrm{S}$ bound to Tr per VP* was diminished by $\sim 60 \%$ in the membrane preparations of both types of photoreceptor cells owing to the faster desensitization of $\mathrm{MII}^{*}$ by the additional phosphorylation processes. The in vitro elements of the current model well reconstructed time courses of Tr activation for both rods and cones in the presence and absence of ATP (Figure 3A, dashed lines (+ATP) and dotted lines (-ATP)) when corresponding light stimulation intensities (a, 0.0085\% in rods, $b, 0.25 \%$ in cones) were applied. As Tachibanaki et al. [25] suggested, the initial rates of $\operatorname{Tr}$ activation with ATP ( $\sim 52.7 \mathrm{Tr} * / \mathrm{VP} * / \mathrm{s}$ in rods and $\sim 15.8 \mathrm{Tr} * / \mathrm{VP} * / \mathrm{s}$ in cones $)$, were almost the same to those in the absence of ATP for both types of photoreceptors $(\sim 52.7$ $\mathrm{Tr} * / \mathrm{VP} * / \mathrm{s}$ in rods and $\sim 15.8 \mathrm{Tr} * / \mathrm{VP} * / \mathrm{s}$ in cones), indicating that the molecular reactions involved in $\mathrm{VP}^{*}$ phosphorylation progressed relatively slowly compared to those of $\mathrm{Tr}$ activation.

Tachibanaki and colleagues have also reported light-induced $\operatorname{Tr}$ activation at different flash intensities (Figure $3 B$ ) in membrane preparations of rods (a, circle) and cones ( $b$, circle) in the presence (filled symbols) and absence (open symbols) of ATP (100 $\mu \mathrm{M})$ [25]. The half-maximal flash intensity for the activation of $\mathrm{Tr}$ was approximately 100 -fold higher in cones than it was in rods. It was also evident that ATP reduced the light sensitivity of $\operatorname{Tr}$ activation in both rods and cones, reflecting the facilitated desensitization of $\mathrm{MII}_{0}{ }_{0}$ through phosphorylation processes. The model also well 
490

491

492

493

494

495

496

497

498

499

500

501

502

503

504

505

506

507

508

509

510

511

512

513

514

515

516

517

518

519

520

521

522

523

524

525

simulated light intensity-dependent Tr activation in both rods and cones. Thus, with the rate constants for the Tr activation reactions $\left(k_{\mathrm{G} 1,0}-k_{\mathrm{G} 1,3}\right.$ and $\left.k_{\mathrm{G} 2}-k_{\mathrm{G} 6}\right)$ and the reaction rates for the VP phosphorylation and inactivation processes with and without ATP in both types of photoreceptors, the model provided reasonable descriptions of the experimental data.

\section{Hydrolysis of cGMP by PDE}

The catalytic activity of PDE in hydrolyzing cGMP increases when the gamma inhibitory subunit is removed from the enzyme upon binding $\operatorname{Tr}^{*}\left(G_{\alpha}-G T P\right)$. The PDE activity thus decreases as $\mathrm{G}_{\alpha}$-GTP is hydrolyzed by its GTPase activity. Koshitani et al. [30] reported time courses of cGMP hydrolysis in membrane preparations of rods and cones in response to light stimulations (Figre $4 A, 0.024 \%$ for rods (a, open circle) and $0.46 \%$ for cones ( $b$, open circle)) in the presence and absence of ATP (solid and dashed lines, respectively) with GTP $\gamma \mathrm{S}$ (filled symbols) and GTP (open symbols). In rods, the number of cGMP hydrolyzed per activated $\mathrm{VP}^{*}$ monotonically increased with GTP $\gamma \mathrm{S}$ increases, in either the presence or absence of ATP. On the other hand, cGMP hydrolyzed with ATP and GTP reached saturation at $\sim 1 / 3$ of that with GTP $\gamma$ S at 50 sec after the light stimulus due to termination of PDE activity as GTP hydrolysis preceded. In contrast, cGMP hydrolysis measured with ATP and GTP $\gamma \mathrm{S}$ in cones was diminished by $\sim 99 \%$ relative to that in rods and was further reduced to $\sim 1 / 6$ with GTP, 25 sec after the light stimulus. The lifetime of Tr was thus estimated to be $\sim 2$-fold shorter in cones [30].

Peak PDE activities (first derivatives of the number of cGMPs hydrolyzed per activated $\mathrm{VP}^{*}$ molecule) were also measured at various intensities of light stimulation (Figure $4 \mathrm{Ba}$, circle for rods and triangle for cones) in the presence of ATP and either GTP (open symbols) or GTP $\gamma \mathrm{S}$ (filled symbols). The peak light intensity-dependent PDE activity with GTP (open symbols) and GTP $\gamma \mathrm{S}$ (filled symbols) was not drastically different in rods and cones, indicating that the rate of GTP hydrolysis is slower than that of GTPdependent or GTP $\gamma$ S-dependent activation of PDE. However, the peak PDE activity was significantly more sensitive to light when the phosphorylation of VPs was prohibited, that is, when ATP was absent (the comparison is shown in Figure $4 B b$ to a).

The in vitro elements of the current model well reconstructed the time courses of cGMP hydrolysis in membrane preparations of rods and cones in response to corresponding light stimulation in the presence and absence of ATP with either GTP or GTP $\gamma \mathrm{S}(a, 0.0085 \%$ in rods and $b, 0.25 \%$ in cones; lines in Figure $4 A$ ). The model also 
well simulated light intensity-dependent PDE activities (lines in Figure $4 B$ ) with the rate constants for the Tr activation reactions $\left(k_{\mathrm{G} 1,0}-k_{\mathrm{G} 1,3}\right.$ and $\left.k_{\mathrm{G} 2}-k_{\mathrm{G} 6}\right)$ as well as the reaction rates for VP phosphorylation and inactivation processes with and without ATP in both types of photoreceptors.

\section{Generation of $I_{\mathrm{CNG}}$}

The $[\mathrm{cGMP}]_{\mathrm{i}}$ is determined by the balance between the rate of cGMP production and degradation under physiological conditions. When $[\mathrm{GTP}]_{\mathrm{i}}$ is elevated, $[\mathrm{cGMP}]_{\mathrm{i}}$ and $I_{\mathrm{CNG}}$ increase because GC generates cGMP at higher rates (see the reaction formulae R.8.1 8.2). Kawamura et al. [14] reported $[\mathrm{GTP}]_{\mathrm{i}}$-dependent $I_{\mathrm{CNG}}$ recorded from a truncated outer segment of rods (Figure $5 A$, circle). Physiologically, the basal $[\mathrm{GTP}]_{\mathrm{i}}$ and $[\mathrm{cGMP}]_{\mathrm{i}}$ in the dark are $1 \mathrm{mM}$ and $-2 \mu \mathrm{M}$, respectively, and the corresponding $I_{\mathrm{CNG}}$ are $\sim-4 \mathrm{pA}$ in rods and $-12 \mathrm{pA}$ in cones. Photoreceptor cells are thus slightly depolarized (-40 mV, [4]). Upon stimulation of the visual pigments, a decrease in the concentration of [cGMP] deactivates the $\mathrm{CNG}$ channels, reducing $I_{\mathrm{CNG}}$ to $\sim 0 \mathrm{pA}$ in response to a strong light stimulus. Kawamura and Tachibanaki reported that light-dependent changes in $I_{\mathrm{CNG}}$ recorded from outer segments of rods (Figure $5 B a$, solid lines) and cones (Figure $5 B b$, solid lines) [8]. The intact elements of the current model simulated not only the [GTP $]_{\mathrm{i}^{-}}$ dependent $I_{\mathrm{CNG}}$ in rods in the dark (Figure $5 \mathrm{~A}$, solid line) but also light-dependent $I_{\mathrm{CNG}}$ responses in the outer segments of both types of photoreceptors (Figure $5 \mathrm{Ba}$, rods and $\mathrm{b}$, cones; dotted lines). Note that these cGMP-dependent currents were simulated under voltage clamp condition (see Eq. S83) since the proposed model does not include other membrane current to calculate changes in membrane potentials. It should be emphasized that the initial rising phases of $I_{\mathrm{CNG}}$ responses, especially in cone, in voltage clamp experiments seems to be faster than these recorded under unclamped condition, most likely due to unchanging driving force for $I_{\mathrm{CNG}}[49]$.

\section{Discussion}

Retinal photoreceptor cells, rods and cones, convert photons of light into chemical and electrical signals as the first step in the visual transduction cascade. The chemical processes of the phototransduction system are very similar to each other in these photoreceptors. The light sensitivity and time resolution of the photoresponse in rods, however, are functionally different from those in cones. To quantitatively and systematically investigate how light intensity-dependent photoresponses are divergently 
regulated in rods and cones, a detailed mathematical models of the visual signal transduction system in these photoreceptors was developed on the basis of the Hamer model [40]. The current model successfully reconstructed a wide variety of light intensity-, ATP- and GTP-dependent changes in the concentrations and activities of phosphorylated VPs and activated Trs/PDEs, as well as CNG currents in rods and cones [14] [2] [8] [1] [30] [17] [25] [20] [45], when taking into account the localized molecular environment.

569

Compared to that in rods, the lower light sensitivity in cones was, at least in part, attributed to the lower affinity of the activated VP for Tr given that the concentration, as well as spatial distributions of VP and Tr at membranous disks in these photoreceptor cells, were very similar to each other. In experiments, the expression of cone $\operatorname{Tr}\left(\mathrm{G}_{\alpha}\right.$ subunit) in rods decreased the light sensitivity of rods and the rate of Tr activation [26] [9]. This biochemical study indicated that the molecular nature of the $\operatorname{Tr} \mathrm{G}_{\alpha}$ subunit in cones, not VP, contributes to the lower light sensitivity and response kinetics of Tr. (inactivation) is another pivotal characteristic leading to lower light sensitivity in cones. While, properties of faster VP desensitization also contribute to the higher time resolution for phototransduction in cones. During the inactivation of $\mathrm{MII}^{*}$, the functional intermediate state of inactive MII, MII ${ }^{\mathrm{i}}$, was assumed before MII* undergoing a complete transition to MIII in both types of photoreceptors [22]. Since the transition from MII*0 to MIII was suggested to be an event on the order of a few minutes in vitro [21] [50], considering that the state $\mathrm{MII}^{\mathrm{i}}$ is indispensable to the termination of visual pigment activity: within $\sim 10$ sec in rods and $\sim 1 \mathrm{sec}$ in cones (see Figure $3 A a$ and $b$, respectively). In cones, the current simulation experiments estimated that the rate of MII* inactivation was $\sim 120$-fold faster than that was in rods. The molecular nature of VP in cones (cone opsin), however, was indistinguishable from that in rods (rhodopsin), at least in terms of their light sensitivity in expression systems [26] [9]. The mechanisms underlying the faster inactivation of cone opsin may be due to other environmental factors and need to be further explored in future experimental studies.

592 On the other hand, to reproduce continuing phosphorylation of VP even after complete termination of its activity, the phosphorylation rate constants for $\mathrm{MII}{ }^{\mathrm{i}}\left(k_{\mathrm{RK} 1}^{\mathrm{i}}-k_{\mathrm{RK}}^{\mathrm{i}}\right)$ were assumed to be slower (1/2) than those for MII*.

595 When complete abolition of VP activities, within $\sim 10$ sec after stimulus, PDE-dependent 596 cGMP hydrolysis still progressed for $\sim 30 \mathrm{sec}$ in rods and cones (shown as + ATP/+GTP 597 in Figure $4 A \mathrm{a}$ and $b$, respectively) due to a longer lifetime of $\operatorname{Tr}^{*}$ in the in vitro 
598

599

600

601

602

603

604

605

606

607

608

609

610

611

612

613

614

615

616

617

618

619

620

621

622

623

624

625

626

627

628

629

630

631

632

633

experimental systems. The assumption of faster inactivation of $\operatorname{Tr}^{*}$ by PDE was therefore indispensable to obtain a faster recovery of $I_{\mathrm{CNG}}$, which was recorded in vivo for both types of photoreceptors (Figure 5). In the current study, the mechanism underlying the fast inactivation of $\operatorname{Tr}^{*}$ was attributed to the RGS9-mediated reaction, which was presumed to be intact under physiological conditions. Compared to that in rods, nearly 20 hold higher expression of RGS9 was assumed in cones ( [20], see Table 3); therefore, the higher temporal resolution of the electrical waveforms of the light intensitydependent $I_{\text {CNG }}$ was achieved in these cells (see Figure 5). Note that RGS9-independent degradation of $\operatorname{Tr}^{*}$ was also assumed to be faster in cones due to the expression level of RGS9 [20]. Although RGS9 was simply assumed to be translocated from the membranous disk at the outer segments to the inner segments of photoreceptor cells during sample preparation in vitro experiments [29], different mechanisms may be involved in the distinct time courses of the termination of PDE activity and recovery of $I_{\mathrm{CNG}}$ in both rods and cones.

For the simulation experiments, the molecular environment of the membranous disk, where all the chemical reactions for phototransduction take place to generate $I_{\mathrm{CNG}}$ in vivo (Figure 5), was assumed to be identical to that in the in vitro system (see Figures 2 - 4 and Table 3). This assumption was based on the observations that VP, Tr, PDE, and RK are closely associated with membranous disks even after undergoing isolation procedures for the biochemical experiments [2]. If chemical reactions among freely diffusing factors are assumed, then the time course of phototransduction would be significantly slower due to the apparent reduction in the concentrations of key signaling molecules in the visual transduction cascade. The assumption made for the microdomain of the membranous disk seems to be verified since VP phosphorylation, Tr activation, and cGMP hydrolysis observed at different concentrations of phototransduction factors in different experimental studies were all well reconstructed by the current simulation study.

For the current model, most of the parameters were determined based on the experimental reports as fully described in the Methods or Results section, whereas some parameters were estimated by fitting experimental data. The rate constants for the reactions of $\mathrm{Tr}$ activation, $k_{\mathrm{G} 1,1}-k_{\mathrm{G} 7}$ (see Table 3), were estimated by model fitting to the initial rate of $\operatorname{Tr}$ activation in response to light flash stimulation as well as light intensity-dependent activation of Tr in vitro [25] [20] (see Figure 3). However, the simulation experiments revealed that the time course and the light intensity-dependent 
634 activation of $\mathrm{Tr}$, as shown in Figure 3 , may be reproduced with various sets of $k_{\mathrm{G} 1,0}-k_{\mathrm{G} 7}$ 635 values. Specifically, the higher light sensitivity of rods can be reproduced even when the 636 affinity of the activated VP to Tr ratio is lower than it is in cones when the subsequent 637 molecular reactions $\left(k_{\mathrm{G} 1,1}-k_{\mathrm{G} 7}\right)$ are faster. Since these parameters have not been 638 conclusively determined by experimental studies, $k_{\mathrm{G} 1,1}-k_{\mathrm{G} 7}$ were set by referring to 639 former simulation studies [11] [10] [40]. Furthermore, the $\mathrm{G}_{\alpha}$-GTP-binding rate in MII*0 $640-\mathrm{MII}_{3}{ }_{3}\left(k_{\mathrm{G} 1,0}-k_{\mathrm{G} 1,3}\right)$ was assumed to decrease with successive phosphorylation of the VPs 641 [27]. The reduction rate was determined by the parameter denoted by $\omega$ in R.3.6 (see the 642 Methods section under Activation and Inactivation of Transducins). The current 643 simulation study clarified that the higher the values of $\omega$, the slower the transducin 644 activation (Figure 3) as well as PDE-mediated cGMP hydrolysis (Figure 4) in the 645 presence of ATP. Although the value of $\omega$ for rods was estimated to be 0.6 by Gibson et 646 al. [27], the value for cones was not given in the literature. In this study, $\omega$ was estimated 647 by fitting the experimental data, as shown in Figures 3 and 4, and found to be 0.9 for 648 cones.

649

650 The reproduction of a single photon response [51] has long been considered one of the 651 most important characteristics to reproduce by a mathematical model. Prior theoretical 652 analysis has concluded that the VP phosphorylation process requires multiple steps, and 653 VP affinity for RK and Tr must exponentially decline as VP phosphorylation proceeds 654 [15] [40] [10]. However, these theoretical models failed to reproduce the time courses of 655 VP phosphorylation or $\operatorname{Tr}$ activation observed in the experiments, therefore, some 656 unknown mechanisms must be involved to control these chemical processes. Importantly, 657 the unknown mechanisms do not contradict the current model, since the model well 658 reproduce both the microscopic and macroscopic experimental observations, although 659 the current model failed to reproduce SPR experiments.

660

661 Finally, the uncertain rate for RGS9-mediated inactivation of $\operatorname{Tr}^{*}$ was estimated by 662 fitting the recovery time course of the electrical waveforms of the light intensity663 dependent $I_{\mathrm{CNG}}$, as shown in Figure $5 B$, based on the concentration of RGS9 and the rate 664 constants for GC-mediated cGMP production determined by Tachibanaki et al. [20] and 665 Kawamura and Murakami [14], respectively. Since GC-mediated cGMP production is 666 regulated by $\mathrm{Ca}^{2+}$, the total content of endogenous $\mathrm{Ca}^{2+}$ buffer $(e \mathrm{~T})$ and the rate constants 667 for the reactions of $\mathrm{Ca}^{2+}$ binding to the endogenous buffers ( $k_{b 1}$ and $k_{b 2}$ ) also determine 668 the recovery time course of the light intensity-dependent $I_{\mathrm{CNG}}$. Although the parameters 669 for the endogenous $\mathrm{Ca}^{2+-b u f f e r i n g}$ system estimated in the current study were 
comparable to those in the Hamer model [40], these values need to be re-evaluated if the rate constants for RGS9-mediated inactivation of $\operatorname{Tr}^{*}$ was experimentally determined.

Meanwhile, other chemical reactions, listed below, were purely driven from assumptions due to limited details in literature. These model assumptions would derive new working hypothesis for future experimental studies to clarify corresponding uncertainties.

677 1. During the inactivation of $\mathrm{MII}_{0}^{*}$, intermediate state of inactive MII, MII ${ }^{\mathrm{i}}$, that has no capability to activate $\operatorname{Tr}$, was introduced.

2. Number of VP phosphorylation for the in intact model was restricted to 1 by taking into consideration arrestin binding.

3. Elution of G $\alpha$-GTP, assumed for the in vitro model, was considered negligible for the intact model.

4. Reactions at membranous disk of the in vitro model needed to be evaluated after scaling substrate concentration.

685

686 As described above, the set of parameters used in the current model are not the only 687 definitive values to reproduce experimental results. In order to analyze the significance 688 of model parameters, sensitivity analysis were performed. 28 parameters related with 689 VP phosphorylation, Tr activation and inactivation, PDE activation, GC activity, RGS 690 activity were increased by $5 \%$ and the sum of squared differences between the resulting 691 time courses of $\mathrm{Pi} / \mathrm{VP}^{*}$ and $I_{\mathrm{CNG}}$, and the original time courses were evaluated which are 692 shown in Figure S1 $A$ and $B$ (in Supplement), respectively (results normalized). Figure $693 \mathrm{~S} 1 A$ shows that the phosphorylation process was strongly related to the $k_{\mathrm{RK} 1}-k_{\mathrm{RK} 6}$ 694 parameters, however, especially for cone, they were also related with $\mathrm{Tr}$ activation and 695 inactivation, and PDE activation processes. Similarly, Figure S1 $B$ shows that the $I_{\mathrm{CNG}}$ 696 were strongly related with both phosphorylation, Tr activation and cGMP production 697 processes. To see the detail of the relation between these parameters and the rising phase 698 and the falling phase of $I_{\mathrm{CNG}}$, sensitivity analysis on the maximum and the minimum 699 slope of $I_{\mathrm{CNG}}$ were performed (see Figure $\mathrm{S} 2$ in Supplement). The rising phase was closely 700 related with phosphorylation, Tr activation and PDE activation parameters (Figure S2 701 A), while the falling phase was related with phosphorylation, Tr activation and cGMP 702 production and RGS activities (Figure S2 B). 
706 light stimulation that lasted for only a short period, corresponding to experimental 707 conditions, the cellular responses during light/dark adaptation may not be accurately 708 predicted since the reactions for VP re-formation from opsin and 11-cis-retinal were not 709 included in the systems. For future studies, it will be worthwhile to include the molecular 710 reactions regarding the retinal cycle to predict the adaptation phenomena of the 711 nonlinear behaviors of photoreceptors in response to light stimuli, as they refer to 712 previous conditions.

713 It also should be emphasized that arrestin is another key molecule to determine VP 714 inactivation through binding to phosphorylated VP. Therefore, incorporating arrestin 715 reactions to the current model is fundamental to understand the entire biological process 716 of VP inactivation. However, experimental data available in literature, as far as we were 717 aware, were not sufficient enough to derive the binding rates especially when changes 718 depending on number of phosphorylation of VP. Accordingly, for the current model, 719 macroscopic data fitting was performed for arrestin-dependent VP inactivation, whereas 720 it needs to be updated when sufficient experimental evidences are available in the future. 721 Finally, few parameters, ATP and GTP concentrations and Kc (the calcium 722 concentration of half maximal cGMP production rate) for the current lower vertebrate 723 model were obtained from experimental studies performed on mammals. Although ATP 724 and GTP concentrations do not require temperature conversion or any additional scaling 725 processes, Kc value need to be replaced when available in literature. 
728 Table 1. Parameters of the membrane-associated molecules applied for simulation in comparison to

729 these estimated in the experimental studies. Wet column represents in vitro substrate concentrations

730 calculated after taking into consideration the experimental test tube volume, while Sim. refers to

731 physiological in vivo concentrations. The Sim. values were applied for the simulation experiments

732 assuming that the in vivo membrane structures were preserved in the in vitro experimental

733 environments (see Discussion section for more details).

\begin{tabular}{|c|c|c|c|c|c|c|c|c|}
\hline & & & $\begin{array}{c}\text { VP } \\
(\mu \mathrm{M})\end{array}$ & $\begin{array}{c}\mathrm{RK} \\
(\mu \mathrm{M})\end{array}$ & $\begin{array}{l}\mathrm{PDE} \\
(\mu \mathrm{M})\end{array}$ & $\begin{array}{c}\operatorname{Tr} \\
(\mu \mathrm{M})\end{array}$ & $\begin{array}{l}\text { ATP } \\
(\mu \mathrm{M})\end{array}$ & $\begin{array}{l}\text { GTP } \\
(\mu \mathrm{M})\end{array}$ \\
\hline \multirow{4}{*}{ Phosphorylation } & \multirow{2}{*}{ Wet } & Rod & 0.5 & 0.002 & 0.0018 & 0.047 & 100 & 500 \\
\hline & & Cone & 0.5 & 0.02 & 0.0018 & 0.047 & 100 & 500 \\
\hline & \multirow{2}{*}{ Sim. } & Rod & 3000 & 12 & 11 & 280 & 100 & 500 \\
\hline & & Cone & 3000 & 120 & 11 & 280 & 100 & 500 \\
\hline \multirow{4}{*}{$\begin{array}{c}\operatorname{Tr} \text { activation } \\
\text { (time-dependent) }\end{array}$} & \multirow{2}{*}{ Wet } & Rod & 0.6 & 0.0024 & 0.0022 & 0.056 & 1000 & 100 \\
\hline & & Cone & 0.3 & 0.012 & 0.011 & 0.028 & 1000 & 100 \\
\hline & \multirow{2}{*}{ Sim. } & Rod & 3000 & 12 & 11 & 280 & 1000 & 100 \\
\hline & & Cone & 3000 & 120 & 11 & 280 & 1000 & 100 \\
\hline \multirow{4}{*}{$\begin{array}{c}\text { Tr activation } \\
\text { (light-dependent) }\end{array}$} & \multirow{2}{*}{ Wet } & Rod & 3 & 0.012 & 0.011 & 0.28 & 100 & 5 \\
\hline & & Cone & 0.3 & 0.012 & 0.0011 & 0.028 & 100 & 5 \\
\hline & \multirow{2}{*}{ Sim. } & Rod & 3000 & 12 & 11 & 280 & 100 & 5 \\
\hline & & Cone & 3000 & 120 & 11 & 280 & 100 & 5 \\
\hline \multirow{4}{*}{ PDE activation } & \multirow{2}{*}{ Wet } & Rod & 0.75 & 0.003 & 0.0028 & 0.07 & 250 & 100 \\
\hline & & Cone & 0.75 & 0.03 & 0.0028 & 0.07 & 250 & 100 \\
\hline & \multirow{2}{*}{ Sim. } & Rod & 3000 & 12 & 11 & 280 & 250 & 100 \\
\hline & & Cone & 3000 & 120 & 11 & 280 & 250 & 100 \\
\hline \multirow{4}{*}{ Photocurrent } & \multirow{2}{*}{ Wet } & Rod & 3000 & 12 & 11 & 280 & 1000 & 1000 \\
\hline & & Cone & 3000 & 120 & 11 & 280 & 1000 & 1000 \\
\hline & \multirow{2}{*}{ Sim. } & Rod & 3000 & 12 & 11 & 280 & 1000 & 1000 \\
\hline & & Cone & 3000 & 120 & 11 & 280 & 1000 & 1000 \\
\hline
\end{tabular}




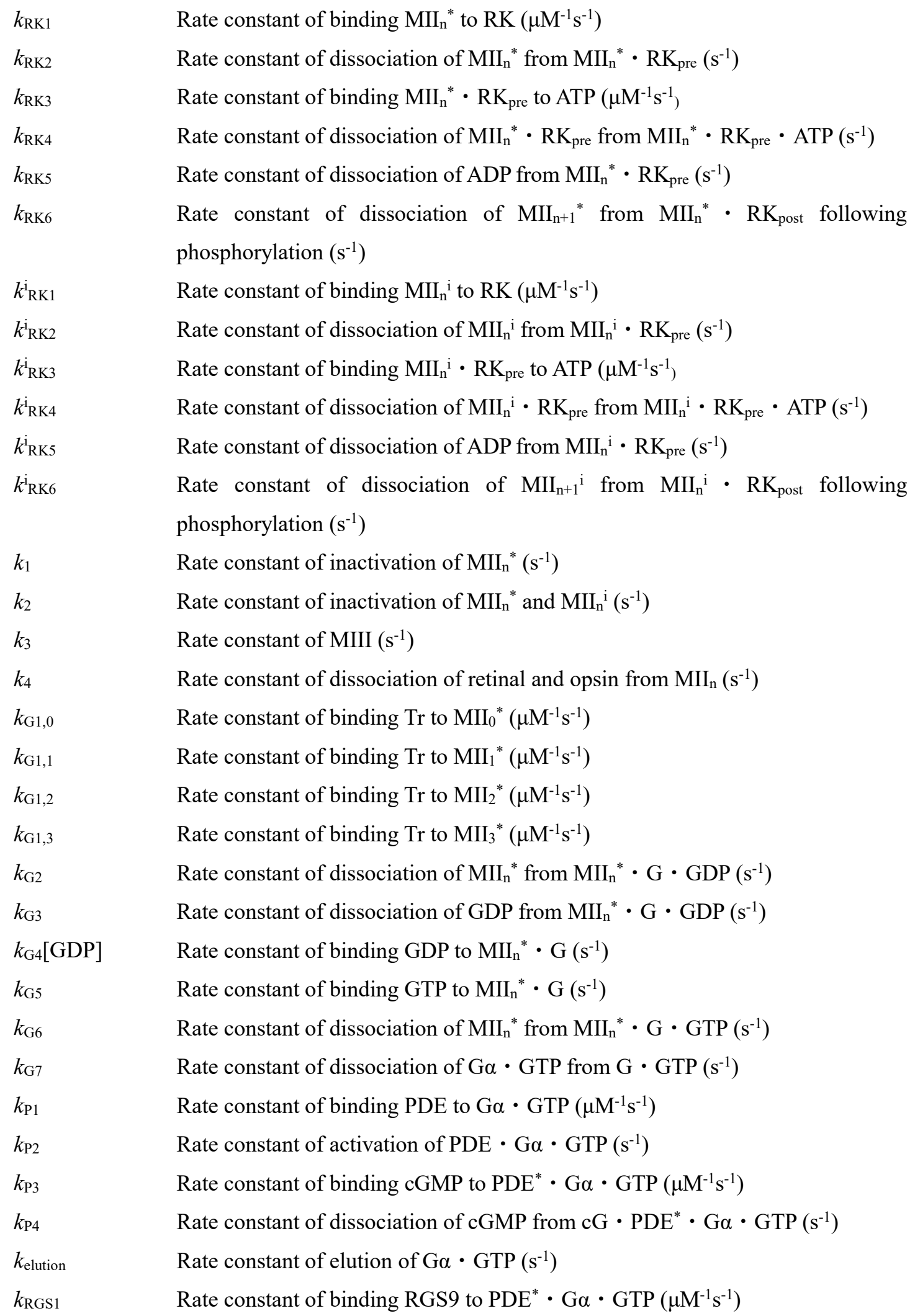




\begin{tabular}{|c|c|}
\hline \multirow{3}{*}{$\begin{array}{l}k_{\mathrm{RGS} 2} \\
k_{\mathrm{RGS} 3}\end{array}$} & Rate constant of dissociation of RGS9 from RGS9 $\cdot \mathrm{PDE}^{*} \cdot \mathrm{G} \alpha \cdot \mathrm{GTP}\left(\mathrm{s}^{-1}\right)$ \\
\hline & Rate constant of dissociation of RGS9 from RGS9 $\cdot \mathrm{PDE}^{*} \cdot \mathrm{G} \alpha \cdot \mathrm{GTP}$ and GTP \\
\hline & hydrolysis $\left(\mathrm{s}^{-1}\right)$ \\
\hline$k_{\text {decay }}$ & Rate constant of inactivation of $\mathrm{G} \alpha \cdot \mathrm{GTP}\left(\mathrm{s}^{-1}\right)$ \\
\hline$k_{\mathrm{GC} 1}$ & Rate constant of binding GC to GTP $\left(\mathrm{s}^{-1}\right)$ \\
\hline$k_{\mathrm{GC} 2}$ & Rate constant of dissociation of cGMP and GC from GC $\cdot \mathrm{GTP}\left(\mathrm{s}^{-1}\right)$ \\
\hline$k_{\mathrm{GC} 3}$ & Rate constant of dissociation of GMP from GC $\cdot$ GTP $\left(\mathrm{s}^{-1}\right)$ \\
\hline$K_{\mathrm{c}}$ & Calcium ion at which synthesis of cGMP is half of maximum rate of cGMP $(\mu \mathrm{M})$ \\
\hline$m$ & Hill coefficient for the action of Calcium ion on cyclase rate (-) \\
\hline$\beta_{\text {dark }}$ & Dark rate of cGMP hydrolysis $\left(\mathrm{s}^{-1}\right)$ \\
\hline$\beta_{\text {sub }}$ & Rate constant of cGMP hydrolysis by cG $\cdot \mathrm{PDE}^{*} \cdot \mathrm{G} \alpha \cdot \mathrm{GTP}\left(\mathrm{s}^{-1}\right)$ \\
\hline$b$ & Ratio of calcium ion to Photocurrent \\
\hline$\gamma_{\mathrm{Ca}}$ & Rate constant of calcium ion extrusion by the $\operatorname{NCKX}\left(\mathrm{s}^{-1}\right)$ \\
\hline$c_{0}$ & Minimum intracellular calcium ion $(\mu \mathrm{M})$ \\
\hline$e_{\mathrm{T}}$ & Concentration calcium ion buffers total $(\mu \mathrm{M})$ \\
\hline$k_{\mathrm{b} 1}$ & Rate constant of binding calcium ion to buffers $\left(\mu \mathrm{M}^{-1} \mathrm{~s}^{-1}\right)$ \\
\hline$k_{\mathrm{b} 2}$ & Rate constant of dissociation of calcium ion from buffers $\left(\mathrm{s}^{-1}\right)$ \\
\hline$K_{\mathrm{m}}$ & Half maximum of cGMP concentration $(\mu \mathrm{M})$ \\
\hline$n_{\mathrm{h}}$ & Hill coefficient for opening CNG channels (-) \\
\hline$F$ & Faraday constant $(\mathrm{C} / \mathrm{mol})$ \\
\hline Vol & Cytoplasmic volume (L) \\
\hline$G_{\max }$ & Conductance of photocurrent (pA/mV) \\
\hline$E_{\mathrm{p}}$ & Reversal potential (mV) \\
\hline$V_{\mathrm{m}}$ & Membrane potential (mV) \\
\hline $\mathrm{VP}_{\text {total }}$ & Concentration of visual pigment total $(\mu \mathrm{M})$ \\
\hline MII $^{*}$ & Concentration of activated metarhodopsin II $(\mu \mathrm{M})$ \\
\hline $\mathrm{MII}^{\mathrm{i}}$ & $\begin{array}{l}\text { Concentration of intermediate state of activated metarhodopsin II and } \\
\text { metarhodopsin III }(\mu \mathrm{M})\end{array}$ \\
\hline $\mathrm{RK}_{\text {free }}$ & Concentration of rhodopsin kinase total $(\mu \mathrm{M})$ \\
\hline $\operatorname{Tr}_{\text {free }}$ & Concentration of transducin total $(\mu \mathrm{M})$ \\
\hline $\mathrm{PDE}_{\text {free }}$ & Concentration of phosphodiesterase $(\mu \mathrm{M})$ \\
\hline $\mathrm{RGS9}_{\text {free }}$ & Concentration of RGS9 free $(\mu \mathrm{M})$ \\
\hline cGMP & Concentratino of cGMP $(\mu \mathrm{M})$ \\
\hline ATP & Concentration of ATP $(\mu \mathrm{M})$ \\
\hline GTP & Concentration of GTP $(\mu \mathrm{M})$ \\
\hline $\mathrm{GC}_{\text {free }}$ & Concentration of Guanylate Cyclase total $(\mu \mathrm{M})$ \\
\hline
\end{tabular}


$\mathrm{Ca}^{2+}$

$\mathrm{Cab}$

$I_{\text {photo }}$
Concentration of calcium ion $(\mu \mathrm{M})$

Concentration of intracellular calcium bound to buffers $(\mu \mathrm{M})$

Photocurrent (pA) 
Table 3. Parameters in the current models

in vitro model

intact model

\begin{tabular}{|c|c|c|c|c|c|c|c|}
\hline Parameter & Unit & Rod & Cone & Rod & Cone & References & Species \\
\hline$k_{\mathrm{RK} 1}$ & $\mu \mathrm{M}^{-1} \mathrm{~s}^{-1}$ & 0.462 & 9.6 & 0.462 & 9.6 & [17] & carp \\
\hline$k_{\mathrm{RK} 2}$ & $\mathrm{~s}^{-1}$ & 6.0 & 80 & 6.0 & 80 & [17] & carp \\
\hline$k_{\mathrm{RK} 3}$ & $\mu \mathrm{M}^{-1} \mathrm{~s}^{-1}$ & 0.16 & 0.26 & 0.16 & 0.26 & [17] & carp \\
\hline$k_{\mathrm{RK} 4}$ & $\mathrm{~s}^{-1}$ & 1.5 & 7.0 & 1.5 & 7.0 & [17] & carp \\
\hline$k_{\mathrm{RK} 5}$ & $\mathrm{~s}^{-1}$ & 1.083 & 37.5 & 1.083 & 37.5 & [17] & carp \\
\hline$k_{\mathrm{RK} 6}$ & $\mathrm{~s}^{-1}$ & 100 & 1500 & 100 & 1500 & [17] & carp \\
\hline$k_{\mathrm{RK} 1}^{\mathrm{i}}$ & $\mu \mathrm{M}^{-1} \mathrm{~s}^{-1}$ & 0.231 & 4.8 & 0.231 & 4.8 & {$[17][20]$} & carp \\
\hline$k_{\mathrm{RK} 2}^{\mathrm{i}}$ & $\mathrm{s}^{-1}$ & 3.0 & 40 & 3.0 & 40 & {$[17][20]$} & carp \\
\hline$k_{\mathrm{RK} 3}^{\mathrm{i}}$ & $\mu \mathrm{M}^{-1} \mathrm{~s}^{-1}$ & 0.08 & 0.13 & 0.08 & 0.13 & {$[17][20]$} & carp \\
\hline$k_{\mathrm{RK} 4}^{\mathrm{i}}$ & $\mathrm{s}^{-1}$ & 0.75 & 3.5 & 0.75 & 3.5 & {$[17][20]$} & carp \\
\hline$k_{\mathrm{RK} 5}^{\mathrm{i}}$ & $\mathrm{s}^{-1}$ & 0.542 & 18.75 & 0.542 & 18.75 & {$[17][20]$} & carp \\
\hline$k_{\mathrm{RK} 6}^{\mathrm{i}}$ & $\mathrm{s}^{-1}$ & 50 & 750 & 50 & 750 & {$[17][20]$} & carp \\
\hline$k_{1}$ & $\mathrm{~s}^{-1}$ & 1.0 & 120 & 1.0 & 120 & [2] & carp \\
\hline$k_{2}$ & $\mathrm{~s}^{-1}$ & 0.0056 & 0.0336 & 0.0056 & 0.0336 & {$[2] \quad[24]$} & carp,frog \\
\hline$k_{3}$ & $\mathrm{~s}^{-1}$ & 0.001 & 0.006 & 0.001 & 0.006 & {$[2] \quad[24]$} & carp,frog \\
\hline$k_{4}$ & $\mathrm{~s}^{-1}$ & 0.0053 & 0.0318 & 0.0053 & 0.0318 & {$[2] \quad[24]$} & carp,frog \\
\hline $\mathrm{k}_{\mathrm{G} 1,0}$ & $\mu \mathrm{M}^{-1} \mathrm{~s}^{-1}$ & 3.57 & 35.7 & 3.57 & 35.7 & {$[10][20]$} & $\begin{array}{l}\text { vertebrate, } \\
\text { carp }\end{array}$ \\
\hline$k_{\mathrm{G} 1,1}$ & $\mu \mathrm{M}^{-1} \mathrm{~s}^{-1}$ & 1.96 & 14.5 & 0 & 0 & [20] & carp \\
\hline$k_{\mathrm{G} 1,2}$ & $\mu \mathrm{M}^{-1} \mathrm{~s}^{-1}$ & 1.08 & 5.9 & 0 & 0 & [20] & carp \\
\hline$k_{\mathrm{G} 1,3}$ & $\mu \mathrm{M}^{-1} \mathrm{~s}^{-1}$ & 0.59 & 2.4 & 0 & 0 & [20] & carp \\
\hline$k_{\mathrm{G} 2}$ & $\mathrm{~s}^{-1}$ & 50 & 2250.34 & 50 & 2250.34 & {$[11][20]$} & $\begin{array}{l}\text { vertebrate, } \\
\text { carp }\end{array}$ \\
\hline$k_{\mathrm{G} 3}$ & $\mathrm{~s}^{-1}$ & 1000 & 370.4 & 1000 & 370.4 & {$[10][20]$} & $\begin{array}{l}\text { vertebrate, } \\
\text { carp }\end{array}$ \\
\hline$k_{\mathrm{G} 4}[\mathrm{GDP}]$ & $\mathrm{s}^{-1}$ & 600 & 2.0 & 600 & 2.0 & {$[10][20]$} & $\begin{array}{l}\text { vertebrate, } \\
\text { carp }\end{array}$ \\
\hline$k_{\mathrm{G} 5}$ & $\mathrm{~s}^{-1}$ & 3.0 & 1 & 3.0 & 1 & {$[10][20]$} & $\begin{array}{l}\text { vertebrate, } \\
\text { carp }\end{array}$ \\
\hline$k_{\mathrm{G} 6}$ & $\mathrm{~s}^{-1}$ & 2000 & 2000 & 2000 & 2000 & [10] & vertebrate \\
\hline$k_{\mathrm{G} 7}$ & $\mathrm{~s}^{-1}$ & 200 & 200 & 200 & 200 & [10] & $\begin{array}{l}\text { vertebrate, } \\
\text { carp }\end{array}$ \\
\hline$k_{\mathrm{P} 1}$ & $\mu \mathrm{M}^{-1} \mathrm{~s}^{-1}$ & 250 & 300 & 250 & 300 & [8] [30] & carp \\
\hline
\end{tabular}




\begin{tabular}{|c|c|c|c|c|c|c|c|}
\hline$k_{\mathrm{P} 2}$ & $\mathrm{~s}^{-1}$ & 1000 & 1000 & 1000 & 1000 & [8] [30] & carp \\
\hline$k_{\mathrm{P} 3}$ & $\mu \mathrm{M}^{-1} \mathrm{~s}^{-1}$ & 100 & 100 & 100 & 100 & [8] [30] & carp \\
\hline$k_{\mathrm{P} 4}$ & $\mathrm{~s}^{-1}$ & 1.0 & 1.0 & 1.0 & 1.0 & [8] [30] & carp \\
\hline$k_{\text {elution }}$ & $\mathrm{s}^{-1}$ & 80000 & 80000 & 0.0 & 0.0 & [8] [30] & carp \\
\hline$k_{\mathrm{RGS} 1}$ & $\mu \mathrm{M}^{-1} \mathrm{~s}^{-1}$ & - & - & 0.85 & 0.7 & {$[8]$} & carp \\
\hline$k_{\mathrm{RGS} 2}$ & $\mathrm{~s}^{-1}$ & - & - & 0.05 & 0.05 & {$[8]$} & carp \\
\hline$k_{\mathrm{RGS} 3}$ & $\mathrm{~s}^{-1}$ & - & - & 5.0 & 4.0 & {$[8]$} & carp \\
\hline$k_{\text {decay }}$ & $\mathrm{s}^{-1}$ & 0.045 & 0.64 & 0.045 & 0.64 & [10] & carp \\
\hline$k_{\mathrm{GC} 1}$ & $\mathrm{~s}^{-1}$ & 0.1 & 0.006 & 0.1 & 0.006 & [14] [45] & frog, carp \\
\hline$k_{\mathrm{GC} 2}$ & $\mathrm{~s}^{-1}$ & 100 & 108.45 & 100 & 108.45 & {$[14][45]$} & frog, carp \\
\hline$k_{\mathrm{GC} 3}$ & $\mathrm{~s}^{-1}$ & 8.5 & 8.967 & 8.5 & 8.967 & {$[14][45]$} & frog, carp \\
\hline$K_{\mathrm{c}}$ & $\mu \mathrm{M}$ & - & - & 0.25 & 0.25 & [13] & bovine \\
\hline$m$ & - & - & - & 2.0 & 2.0 & {$[10]$} & vertebrate \\
\hline$\beta_{\text {dark }}$ & $\mathrm{s}^{-1}$ & - & - & 3.5 & 3.5 & [9] & carp \\
\hline$\beta_{\text {sub }}$ & $\mathrm{s}^{-1}$ & 2000 & 2400 & 2000 & 2400 & {$[2]$} & carp \\
\hline$b$ & $\mu \mathrm{Ms}^{-1} \mathrm{pA}^{-1}$ & - & - & 0.303 & 0.413 & [8] [14] & $\begin{array}{l}\text { carp, } \\
\text { salamander, } \\
\text { bass }\end{array}$ \\
\hline$\gamma_{\mathrm{Ca}}$ & $\mathrm{s}^{-1}$ & - & - & 106.7 & 582.4 & {$[8]$} & carp \\
\hline$c_{0}$ & $\mu \mathrm{M}$ & - & - & 0.01 & 0.01 & [11] & vertebrate \\
\hline$e_{\mathrm{T}}$ & $\mu \mathrm{M}$ & - & - & 400 & 400 & [11] & vertebrate \\
\hline$k_{\mathrm{b} 1}$ & $\mu \mathrm{M}^{-1} \mathrm{~s}^{-1}$ & - & - & 4.0 & 1.0 & {$[8]$} & carp \\
\hline$k_{\mathrm{b} 2}$ & $\mathrm{~s}^{-1}$ & - & - & 16 & 4.0 & {$[8]$} & carp \\
\hline$K_{\mathrm{m}}$ & $\mu \mathrm{M}$ & - & - & 20 & 60 & [8] [45] & carp \\
\hline$n_{\mathrm{h}}$ & - & - & - & 2.0 & 4.0 & [8] [45] & carp \\
\hline $\mathrm{F}$ & $\mathrm{C} / \mathrm{mol}$ & - & - & 96486.7 & 96486.7 & & \\
\hline vol & $\mathrm{L}$ & - & - & $1.2 \mathrm{e}-13$ & $9.0 \mathrm{e}-14$ & {$[8]$} & carp \\
\hline $\mathrm{G}_{\max }$ & $\mathrm{pA} / \mathrm{mV}$ & - & - & 10.1 & 243000.3 & {$[8]$} & carp \\
\hline$K_{\mathrm{p}}$ & $m V^{-1}$ & - & - & 0.03 & 0.015 & {$[8]$} & carp \\
\hline$E_{\mathrm{p}}$ & $\mathrm{mV}$ & - & - & 10 & 10 & {$[52]$} & lizard \\
\hline$V_{\mathrm{m}}$ & $\mathrm{mV}$ & - & - & -30 & -30 & {$[52]$} & lizard \\
\hline $\mathrm{VP}_{\text {total }}$ & $\mu \mathrm{M}$ & 3000 & 3000 & 3000 & 3000 & {$[2]$} & carp \\
\hline $\mathrm{RK}_{\text {free }}$ & $\mu \mathrm{M}$ & 12 & 120 & 12 & 120 & {$[2]$} & carp \\
\hline $\operatorname{Tr}_{\text {free }}$ & $\mu \mathrm{M}$ & 280 & 280 & 280 & 280 & {$[2]$} & carp \\
\hline $\mathrm{PDE}_{\text {free }}$ & $\mu \mathrm{M}$ & 11 & 11 & 11 & 11 & {$[2]$} & carp \\
\hline $\mathrm{RGS}_{\text {free }}$ & $\mu \mathrm{M}$ & - & - & 3.3 & 75 & {$[2]$} & carp \\
\hline
\end{tabular}




\begin{tabular}{|c|c|c|c|c|c|c|c|}
\hline cGMP & $\mu \mathrm{M}$ & - & - & 2.0 & 2.0 & [53] & triturus \\
\hline \multirow[t]{5}{*}{ ATP } & $\mu \mathrm{M}$ & Depen & Depende & 1000 & 1000 & {$[54][55]$} & rabbit, frog, \\
\hline & & dence & nce on & & & {$[56] \quad[57]$} & bovine \\
\hline & & on & experim & & & [58] [59] & \\
\hline & & experi & ent & & & & \\
\hline & & ment & & & & & \\
\hline \multirow[t]{5}{*}{ GTP } & $\mu \mathrm{M}$ & Depen & Depende & 1000 & 1000 & [54] [58] & rabbit, frog \\
\hline & & dence & nce on & & & & \\
\hline & & on & experim & & & & \\
\hline & & experi & ent & & & & \\
\hline & & ment & & & & & \\
\hline $\mathrm{GC}_{\text {free }}$ & $\mu \mathrm{M}$ & 4.2 & 72 & 4.2 & 72 & {$[45]$} & carp \\
\hline $\mathrm{Ca}^{2+}$ & $\mu \mathrm{M}$ & - & - & 0.5 & 0.5 & {$[10]$} & vertebrate \\
\hline $\mathrm{Cab}$ & $\mu \mathrm{M}$ & - & - & 44.4 & 44.4 & [8] & carp \\
\hline$I_{\text {photo }}$ & $\mathrm{pA}$ & - & - & -4 & -12 & [2] & carp \\
\hline
\end{tabular}


742 Table 4. Initial set of time-dependent variables

$\begin{array}{lll} & \text { Rod } & \text { Cone } \\ \mathrm{VP}_{\text {free }} & 3000 & 3000 \\ \mathrm{RK}_{\text {free }} & 12 & 120 \\ \mathrm{PDE}_{\text {free }} & 11 & 11 \\ \mathrm{~T}_{\text {free }} & 280 & 280 \\ \mathrm{RGS9}_{\text {free }} & 3.3 & 75 \\ \mathrm{GC}_{\text {free }} & 8.3 & 75.9 \\ \mathrm{ATP} & 1000 & 1000 \\ \mathrm{GTP} & 1000 & 1000 \\ \mathrm{cGMP} & 2.0 & 2.0 \\ \mathrm{GC} \cdot \mathrm{GTP} & 4.11 & 3.90 \\ \mathrm{Ca}{ }^{2+} & 0.5 & 0.5 \\ \mathrm{Cab} & 44.4 & 44.4 \\ I_{\text {photo }} & -4.0 & -12.0\end{array}$




\section{Figure Legends}

Figure 1 Reaction scheme of visual phototransduction in rods and cones

A, Visual phototransduction, including activation and inactivation of VP, Tr, and PDE, in rods and cones (see Table 2 for abbreviations). B, Details of the phosphorylation reactions. Phosphorylation of VP at 3 sites in vitro (Figures 2-4), where only 1 site in vivo (Figure 5) was assumed. Phosphorylation reactions and Tr* elution indicated in light gray were not included, whereas RGS9-dependent inactivation of Tr* and GC-dependent cGMP synthesis, depicted in dark gray, were added for simulating $I_{\mathrm{CNG}}$ in Figure 5.

Figure 2 Phosphorylation of visual pigments in rods and cones.

A, The time courses for the phosphorylation of VPs (the number of phosphate groups incorporated into an activated visual pigment molecule) measured in the membrane preparations of purified frog rod (a, circle) and carp cone (b, triangle) in response to light flash at $1.3 \%$ and $2.5 \%$, respectively, in the experiments [17]. The corresponding simulation results (dotted lines in a and b) are also shown in the figures. B, Maximum rates of phosphorylation reaction per activated visual pigment at different flash intensities in rods ( $\mathrm{a}$, circle) and cones (b, circle), determined $10 \mathrm{sec}$ and $0.6 \mathrm{sec}$ after light stimuli, respectively (data modified from Tachibanaki et al. [17]). Experimental results were fitted by the Michaelis-Menten equation (V/S = Vmax/(S + Km), solid lines). Simulated responses for both the carp rod ( $a$, dotted line triangle) and cone (b, dotted

A, The time courses for $\operatorname{Tr}$ activation (the number of GTP $\gamma \mathrm{S}$ molecule, a nonhydrolyzable GTP analog, incorporated per VP*) in the membrane preparations of purified carp rods and cones in response to light stimulation $(0.0085 \%$ for rods (a, circle) and $0.25 \%$ for cones (b, circle)) in the presence (filled symbols) and absence (open symbols) of ATP (1 mM) as determined by biochemical experiments [20]. Corresponding simulation results (dotted lines (-ATP) and dashed lines (+ATP) in a and b were 
function of flash intensity in rods (a, circle, after $40 \mathrm{sec}$ stimulation) and cones (b, circle, after $20 \mathrm{sec}$ stimulation) in the presence (filled symbols) and absence (open symbols) of ATP (0.1 mM) normalized to maximum values. Simulated results for rods (a, triangle) and cones ( $b$, triangle) reproduced under corresponding experimental conditions in the presence (filled symbols) and absence (open symbols) of ATP are also shown in the figure. Values for the rate constants for VP phosphorylation, kRK1 - kRK6 and kiRK1 - kiRK6, were set to 0 for simulation of experimental results obtained without ATP.

787

Figure 4 PDE-mediated cGMP hydrolysis in rods and cones

789

A, The time courses for PDE activities (the number of cGMP molecules hydrolyzed per $\mathrm{VP}^{*}$ ) in the membrane of carp rods and cones in response to light stimulations (a, $0.024 \%$ for rods (open circle); b, 0.46\% for cones (open circle)) in the presence of ATP (0.25 $\mathrm{mM})$, cGMP (2500 $\mu \mathrm{M})$ and either GTP (open closed circle) or GTP $\gamma \mathrm{S}$ (filled triangle) determined by biochemical experiments [25] [19]). Corresponding simulation results (solid lines (with GTP $\gamma \mathrm{S}$ ) and dotted lines (with GTP) in a and b) were superimposed onto the experimental data. The continuous presence of saturating levels of cGMP was assumed for the simulation study, and thus $\beta$ dark was set to 0 . B, Light-induced peak PDE activity as a function of flash intensity in rods (circle) and cones (triangle) either in the presence of ATP (a, $0.25 \mathrm{mM}$ ) or without ATP (b) and either GTP (open symbols) or GTP $\gamma$ S (filled symbols) are normalized to maximum values. Simulated PDE activity of the rods and cones reproduced under the corresponding experimental conditions (with either GTP (dotted lines) or GTP $\gamma \mathrm{S}$ (solid lines)) were superimposed onto the experimental results. The value for the rate constant, $k_{\text {decay }}$, for cGMP hydrolysis was set to 0 for the simulation of the experimental results obtained with GTP $\gamma \mathrm{S}$.

A, $[\mathrm{GTP}]_{\mathrm{i}}$-dependent $I_{\mathrm{CNG}}$ recorded from a truncated outer segment of frog rods 810 steady-state were superimposed onto the experimental data (solid line). B, Time courses 811 of light-induced $I_{\mathrm{CNG}}$ as a function of flash intensity recorded from the outer segments of 812 the carp rods (a, light stimuli; 5.1E-6, 1.6E-5, 5.1E-5, 1.6E-4, and 5.1E-4\%) and cones (b, 813 light stimuli; $1.6 \mathrm{E}-3,5.2 \mathrm{E}-3,1.6 \mathrm{E}-2$, and $0.16 \%)$ as solid lines [14]. Simulated $I_{\mathrm{CNG}}$ for

814 rods (a) and cones (b) reproduced under the corresponding experimental conditions was 815 superimposed onto the experimental results (dotted lines in a and b). 
[1] S. Kawamura and S. Tachibanaki, "Explaining the functional differences of rods versus cones," WIRES Membr. Transp. Signal, vol. 1, pp. 675-683, 2012.

[2] S. Kawamura and S. Tachibanaki, Phototransduction in rods and cones. In Vertebrate photoreceptors, T. Furukawa, J. Hurley and S. Kawamura, Eds., Japan: Springer, 2014, pp. 23-45.

[3] E. E. Fesenko, S. S. Kolesnikov and A. L. Lyubarsky, "Induction by cyclic GMP of cationic conductance in plasma membrane of retinal rod outer segment," Nature, vol. 313, pp. 310-313, 1985.

[4] D. A. Baylor, "Photoreceptor signals and vision, Proctor lecture," in Invest Ophthalmol. Vis. Sci., 1987.

[5] M. Tachibana and A. Kaneko, "L-glutamate-induced depolarization in solitary photoreceptors: a process that may contribute to the interaction between photoreceptors in situ," Proc. Natl. Acad. Sci. U. S. A., vol. 85, pp. 5315-5319, 1988.

[6] F. S. Werblin and J. E. Dowling, "Organization of the retina of the mudpuppy, Necturus maculosus. II. Intracellular recording," J. Neurophysiol., vol. 32, pp. 339$355,1969$.

[7] D. Purves, G. Augustine, D. Fitzpatrick and W. Hall, Eds., Vision: The Eye. In Neuroscience, fifth edition, Sinauer associates, Inc., Sunderland, Massachusetts U.S.A., 2012, pp. 229-256.

[8] S. Kawamura and S. Tachibanaki, "Rod and cone photoreceptors: molecular basis of the difference in their physiology," Comp Biochem. Physiol A Mol. Integr. Physiol, vol. 150, pp. 369-377, 2008.

[9] N. Ingram, A. Sampath and G. Fain, "Why are rods more sensitive than cones?," Journal Physiology, vol. 594, no. 19, pp. 5415-5426, 2016.

[10] R. D. Hamer, S. C. Nicholas, D. Tranchina, T. D. Lamb and T. D. Jarvinen, "Toward a unified model of vertebrate rod phototransduction," Vis. Neurosci., vol. 22, pp. 417-436, 2005.

[11] D. Dell'Orco, H. Schmidt, S. Mariani and F. Fanelli, "Network-level analysis of light adaptation in rod cells under normal and altered conditions," Mol. Biosyst., vol. 5, pp. 1232-1246, 2009.

[12] H. Ripps, "Night blindness revisited: from man to molecules," Invest. Ophtalmol. Vis. Sci., vol. 23, pp. 588-609, 1982. 
[13] I. Audo, A. Robson, G. Holder and A. Moore, "The Negative ERG: Clinical Phenotypes and Disease Mechanisms of Inner Retinal Dysfunction," Surv. Ophtalmol., vol. 53, pp. 16-40, 2008.

[14] S. Kawamura and M. Murakami, "Regulation of cGMP levels by guanylate cyclase in truncated frog rod outer segments," J. Gen. Physiol., vol. 94, pp. 649-668, 1989.

[15] F. Rieke and D. A. Baylor, "Origin of reproducibility in the responses of retinal rods to single photons," Biophys. J., vol. 75, pp. 1836-1857, 1998.

[16] N. Sarai, S. Matsuoka and A. Noma, "simBio: A Java package for the," Prog. Biophys. Mol. Bio., vol. 90, pp. 360-377, 2006.

[17] S. Tachibanaki, D. Arinobu, Y. Shimauchi-Matsukawa, S. Tsushima and S. Kawamura, "Highly effective phosphorylation by G protein-coupled receptor kinase 7 of light-activated visual pigment in cones," Proc. Natl. Acad. Sci. U. S. A., vol. 102, pp. 9329-9334, 2005.

[18] A. Mendez, M. E. Burns, A. Roca, J. Lem, L. W. Wu, M. I. Simon, D. A. Baylor and J. Chen, "Rapid and reproducible deactivation of rhodopsin requires multiple phosphorylation sites," Neuron, vol. 28, pp. 153-164, 2000.

[19] U. Wilden and H. Kuhn, "Light-dependent phosphorylation of rhodopsin: number of phosphorylation sites," Biochemistry, vol. 21, pp. 3014-3022, 1982.

[20] S. Tachibanaki, S. Yonetsu, S. Fukaya, Y. Koshitani and S. Kawamura, "Low activation and fast inactivation of transducin in carp cones," J. Biol. Chem., vol. 287, pp. 41186-41194, 2012.

[21] C. Baumann, "Kinetics of slow thermal reactions during the bleaching of rhodopsin in the perfused frog retina," J. Physiol., vol. 222, pp. 643-663, 1972.

[22] T. Ebrey, The Bleaching of Visual Pigments. In CRC Handbook of Organic Photochemistry and Photobiology, W. Horspool and F. Lenci, Eds., CRC PRESS, 2003, pp. 126-1 - 126-9.

[23] W. G., "The molecular basis of visual excitation," Nature, vol. 219, pp. 800-807, 1968.

[24] A. V. Kolesnikov, E. Y. Golobokova and V. I. Govardovskii, "The identity of metarhodopsin III," Vis. Neurosci., vol. 20, pp. 249-265, 2003.

[25] S. Tachibanaki, S. Tsushima and S. Kawamura, "Low amplification and fast visual pigment phosphorylation as mechanisms characterizing cone photoresponses," Proc. Natl. Acad. Sci. U. S. A., vol. 98, pp. 14044-14049, 2001.

[26] C. K. Chen, M. L. Woodruff, F. S. Chen, H. Shim, M. C. Cilluffo and G. L. Fain, 
"Replacing the rod with the cone transducin alpha subunit decreases sensitivity and accelerates response decay," Journal Physiology, vol. 588, no. 17, pp. 3231-3241, 2010.

[27] S. K. Gibson, J. H. Parkes and P. A. Liebman, "Phosphorylation modulates the affinity of light-activated rhodopsin for G protein and arrestin," Biochemistry, vol. 39, pp. 5738-5749, 2000.

[28] N. P. Skiba, J. A. Hopp and V. Y. Arshavsky, "The effector enzyme regulates the duration of $\mathrm{G}$ protein signaling in vertebrate photoreceptors by increasing the affinity between transducin and RGS protein," J. Biol. Chem., vol. 275, pp. 3271632720, 2000.

[29] M. Tian, M. Zallocchi, W. Wang, C. K. Chen, K. Palczewski, D. Delimont, D. Cosgrove and Y. W. Peng, "Light-induced translocation of RGS9-1 and Gbeta5L in mouse rod photoreceptors," PLoS One, vol. 8, p. e58832, 2013.

[30] Y. Koshitani, S. Tachibanaki and S. Kawamura, "Quantitative aspects of cGMP phosphodiesterase activation in carp rods and cones," J. Biol. Chem., vol. 289, pp. 2651-2657, 2014.

[31] A. I. Ames, "Steady state feedback in mammalian phototransduction illustrated by a nomogram," Vision Res., vol. 34, pp. 821-827, 1994.

[32] Y. Koutalos, K. Nakatani, T. Tamura and K. W. Yau, "Characterization of guanylate cyclase activity in single retinal rod outer segments," J. Gen. Physiol., vol. 106, pp. 863-890, 1995.

[33] J. L. Miller and J. I. Korenbrot, "Differences in calcium homeostasis between retinal rod and cone photoreceptors revealed by the effects of voltage on the cGMPgated conductance in intact cells," J. Gen. Physiol., vol. 104, pp. 909-940, 1994.

[34] E. N. Pugh, T. Duda, A. Sitaramayya and R. K. Sharma, "Photoreceptor guanylate cyclases: a review," Biosci. Rep., vol. 17, pp. 429-473, 1997.

[35] E. N. Pugh and T. D. Lamb, "Amplification and kinetics of the activation steps in phototransduction," Biochim. Biophys. Acta, vol. 1141, pp. 111-149, 1993.

[36] J. I. Korenbrot and D. L. Miller, "Cytoplasmic free calcium concentration in darkadapted retinal rod outer segments," Vision Res., vol. 29, pp. 939-948, 1989.

[37] L. Lagnado, L. Cervetto and P. A. McNaughton, "Calcium homeostasis in the outer segments of retinal rods from the tiger salamander," J. Physiol., vol. 455, pp. 111$142,1992$.

[38] A. P. Sampath, H. R. Matthews, M. C. Cornwall and G. L. Fain, "Bleached pigment 
produces a maintained decrease in outer segment $\mathrm{Ca} 2+$ in salamander rods," $J$. Gen. Physiol., vol. 111, pp. 53-64, 1998.

[39] R. D. Hamer, "Computational analysis of vertebrate phototransduction: combined quantitative and qualitative modeling of dark- and light-adapted responses in amphibian rods," Vis. Neurosci., vol. 17, pp. 679-699, 2000.

[40] R. D. Hamer, S. C. Nicholas, D. Tranchina, P. A. Liebman and T. D. Lamb, "Multiple steps of phosphorylation of activated rhodopsin can account for the reproducibility of vertebrate rod single-photon responses," J. Gen. Physiol, vol. 122, pp. 419-444, 2003.

[41] S. Nikonov, N. Engheta and E. N. Pugh, "Kinetics of recovery of the dark-adapted salamander rod photoresponse," J. Gen. Physiol., vol. 111, pp. 7-37, 1998.

[42] K. Nakatani, Y. Koutalos and K. W. Yau, "Ca2+ modulation of the cGMP-gated channel of bullfrog retinal rod photoreceptors," J. Physiol., vol. 484, no. Pt 1, pp. 69-76, 1995.

[43] A. Picones and J. I. Korenbrot, "Analysis of fluctuations in the cGMP-dependent currents of cone photoreceptor outer segments," Biophys. J., vol. 66, pp. 360-365, 1994.

[44] M. S. Sagoo and L. Lagnado, "The action of cytoplasmic calcium on the cGMPactivated channel in salamander rod photoreceptors," J. Physiol, vol. 497 (Pt2), pp. 309-319, 1996.

[45] N. Takemoto, S. Tachibanaki and S. Kawamura, "High cGMP synthetic activity in carp cones," Proc. Natl. Acad. Sci. U. S. A., vol. 106, pp. 11788-11793, 2009.

[46] Cork, K. M., M. J. Van Hook and W. B. Thoreson, "Mechanisms, pools, and sites of spontaneous vesicle release at synapses of rod and cone photoreceptors," Eur. J. Neurosci., vol. 44, pp. 2015-2027, 2016.

[47] F. J. Vinberg, S. Strandman and A. Koskelainen, "Origin of the fast negative ERG component from isolated aspartate-treated mouse retina," J. Vis., vol. 9, pp. 9-17, 2009.

[48] D. Krizaj and D. R. Copenhagen, "Calcium regulation in photoreceptors," Front Biosci., vol. 7, pp. d2023-d2024, 2002.

[49] R. J. Perry and P. A. McNaughton, "RESPONSE PROPERTIES OF CONES FROM THE RETINA OF THE TIGER SALAMANDER," Journal of Physiology, vol. 433, pp. 561-587, 1991.

[50] J. Kibelbek, D. C. Mitchell, J. M. Beach and B. J. Litman, "Functional equivalence 
of metarhodopsin II and the Gt-activating form of photolyzed bovine rhodopsin," Biochemistry, vol. 30, pp. 6761-6768, 1991.

[51] A. Baylor, T. Lamb and K. Yau, "RESPONSES OF RETINAL RODS TO SINGLE PHOTONS," J. Physiol., vol. 288, pp. 613-634, 1979.

[52] Y. Kamiyama, T. Ogura and S. Usui, "Ionic current model of the vertebrate rod photoreceptor," Vision Research, vol. 36, pp. 4059-4068, 1996.

[53] S. Forti, A. Menini, G. Rispoli and V. Torre, "Kinetics of phototransduction in retinal rods of the newt Triturus cristatus," J. Physiol., vol. 419, pp. 265-295, 1989.

[54] S. J. Berger, G. W. DeVries, J. G. Carter, D. W. Shulz, P. N. Passonneau, O. H. Lowry and J. A. Ferrendelli, "The distribution of the components of the cylclic GMP cycle in retina," J. Biol. Chem., vol. 255, pp. 3128-3133, 1980.

[55] M. S. Biernbaum and M. D. Bownds, "Light-induced changes in GTP and ATP in frog rod photoreceptors. Comparison with recovery of dark current and light sensitivity during dark adaptation," J. Gen. Physiol., vol. 85, pp. 107-121, 1985.

[56] W. Hemmer, I. Riesinger, T. Wallimann, H. M. Eppenberger and A. F. Quest, "Braintype creatine kinase in photoreceptor cell outer segments: role of a phosphocreatine circuuit in outer segment energy metabolism and photortransduction," J. Cell. Sci., vol. 106(Pt 2), pp. 671-683, 1993.

[57] L. Notari, I. M. Pepe, C. Cugnoli and A. Morelli, "Adenylate kinase activity in rod outer segments of bovine retina," Biochem. Biophys. Acta, vol. 1504, pp. 438-443, 2001.

[58] W. E. Robinson and W. A. Hagins, "GTP hydrolysis in intact rod outer segments and the transmitter cycle in visual excitation," Nature, vol. 280, pp. 398-400, 1979.

[59] R. G. Zuckerman, J. Schmidt and S. M. Dacko, "Rhodopsin-to-metarhodopsin II transition triggers amplified changes in cytosol ATP and ADP in intact retinal rod outer segments," Proc. Natl. Acad. Sci., vol. 79, pp. 6414-6418, 1982. 


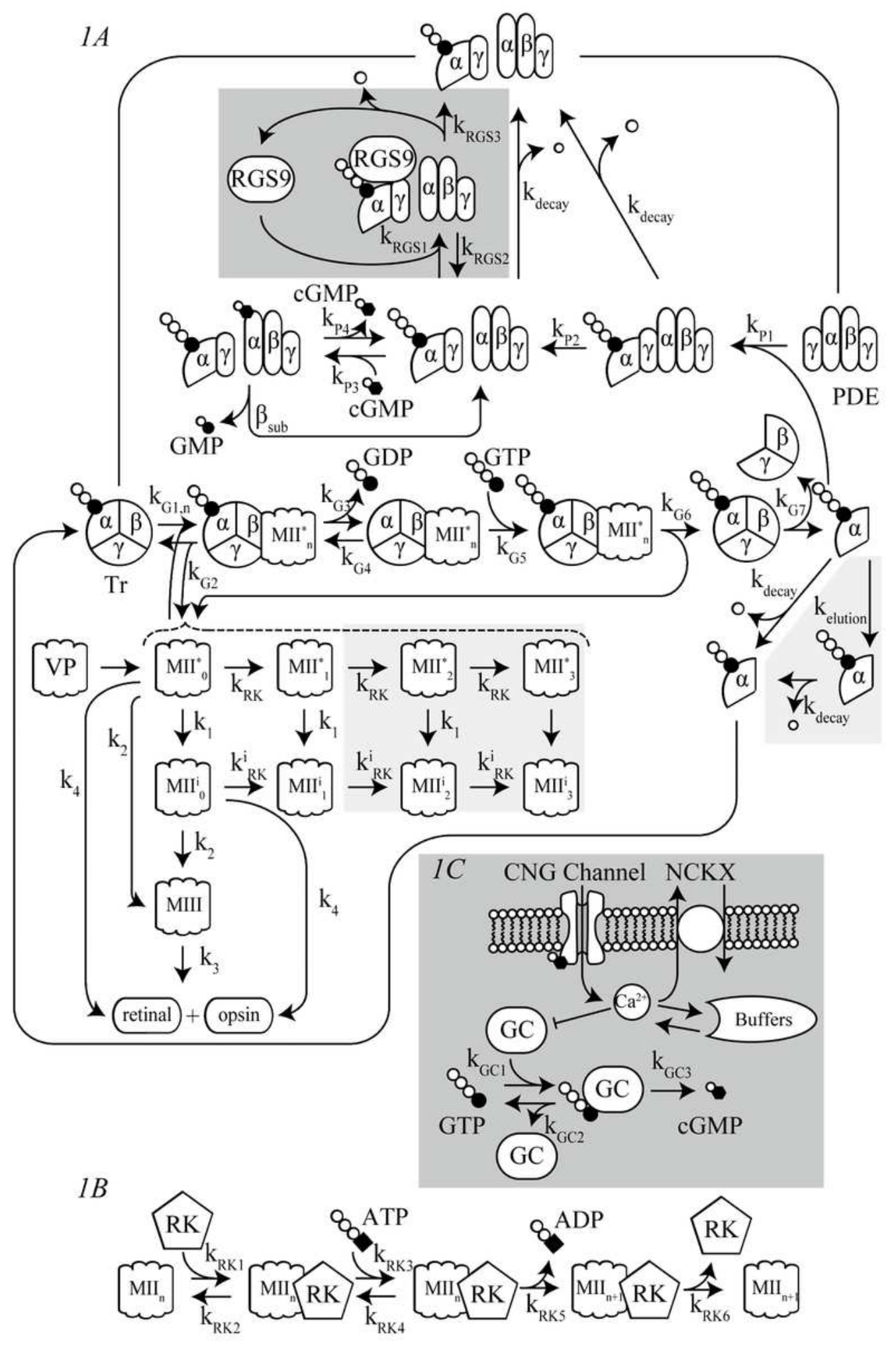

Figure 1

Reaction scheme of visual phototransduction in rods and cones A, Visual phototransduction, including activation and inactivation of VP, Tr, and PDE, in rods and cones (see Table 2 for abbreviations). B, Details of the phosphorylation reactions. Phosphorylation of VP at 3 sites in vitro (Figures 2-4), where only 1 site 
in vivo (Figure 5) was assumed. Phosphorylation reactions and Tr* elution indicated in light gray were not included, whereas RGS9-dependent inactivation of Tr* and GC-dependent cGMP synthesis, depicted in dark gray, were added for simulating ICNG in Figure 5.
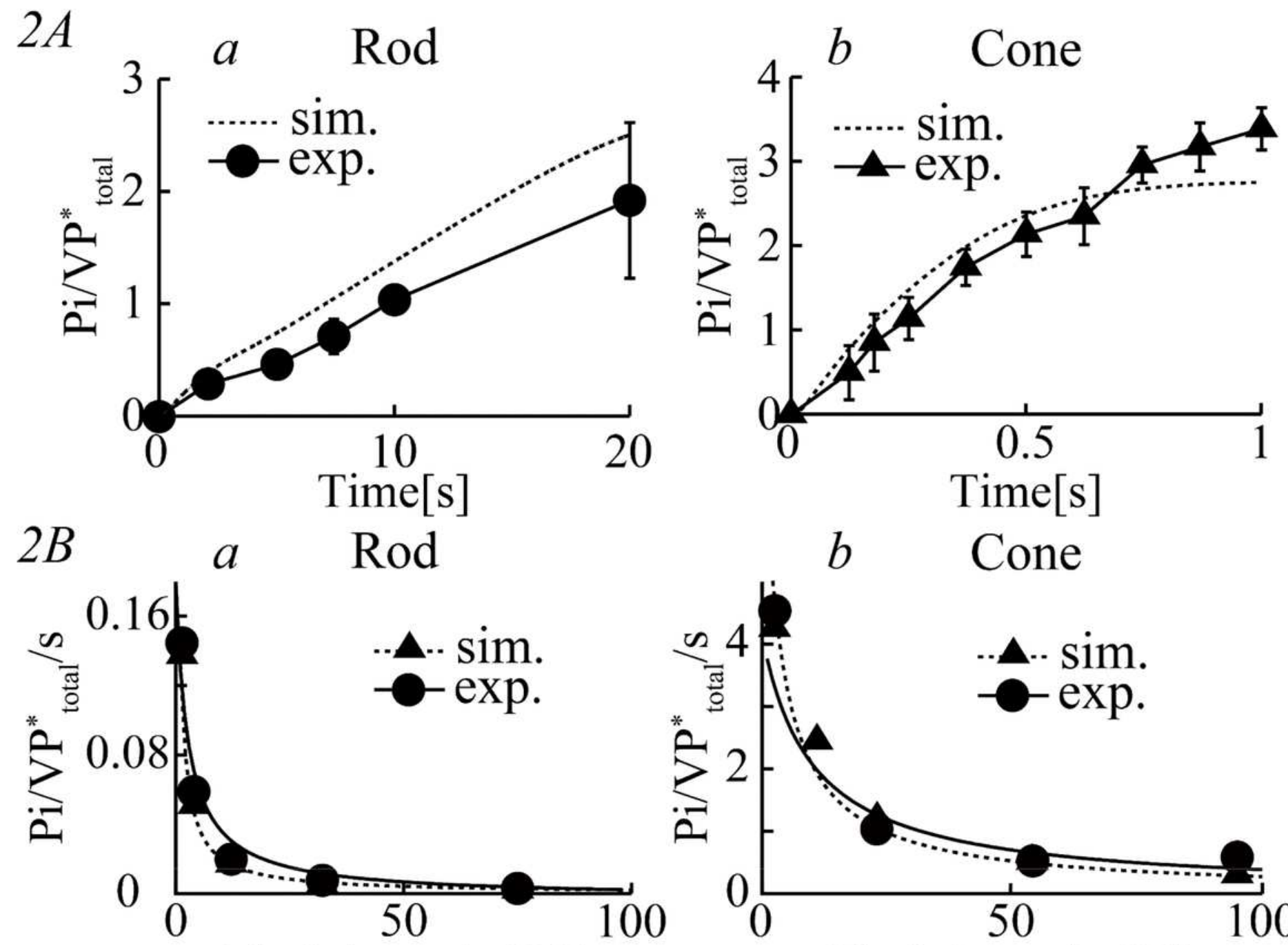

Flash intensity[\%]

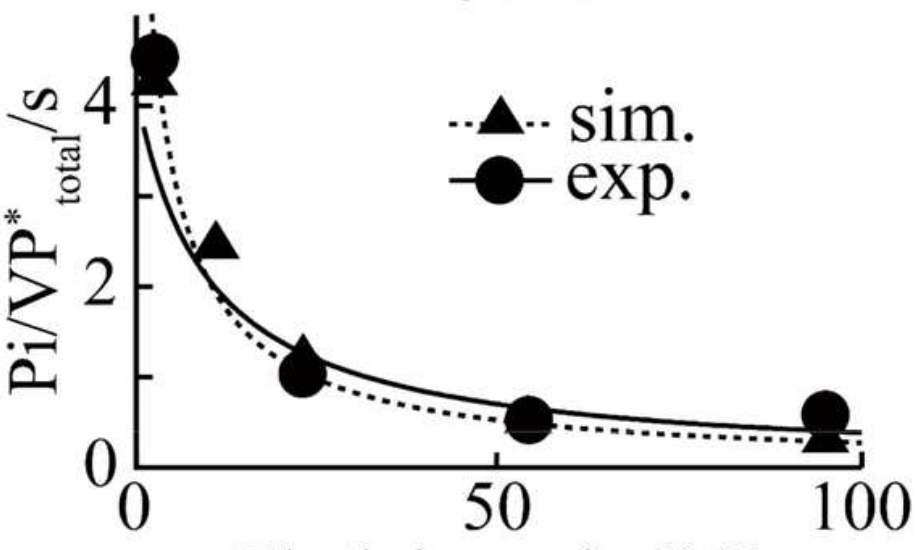

Flash intensity[\%]

Figure 2

Phosphorylation of visual pigments in rods and cones. A, The time courses for the phosphorylation of VPs (the number of phosphate groups incorporated into an activated visual pigment molecule) measured in the membrane preparations of purified frog rod ( $a$, circle) and carp cone (b, triangle) in response to light flash at $1.3 \%$ and $2.5 \%$, respectively, in the experiments [17]. The corresponding simulation results (dotted lines in a and b) are also shown in the figures. B, Maximum rates of phosphorylation reaction per activated visual pigment at different flash intensities in rods (a, circle) and cones (b, circle), determined $10 \mathrm{sec}$ and $0.6 \mathrm{sec}$ after light stimuli, respectively (data modified from Tachibanaki et al. [17]).

Experimental results were fitted by the Michaelis-Menten equation (V/S = Vmax/(S + Km), solid lines). Simulated responses for both the carp rod (a, dotted line triangle) and cone (b, dotted line triangle) were superimposed onto the experimental results. 

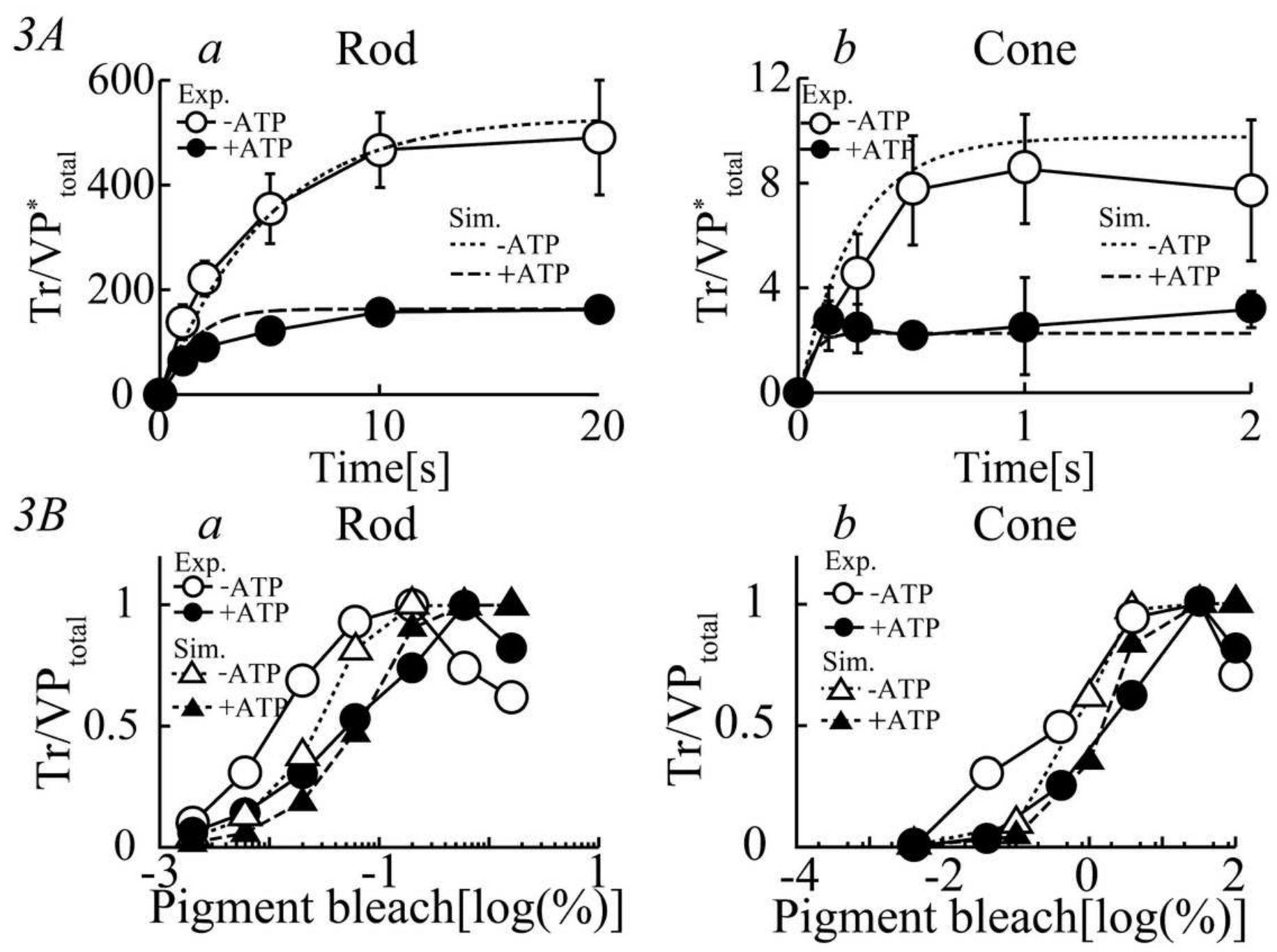

Figure 3

Transducin activation in rods and cones A, The time courses for Tr activation (the number of GTPYS molecule, a nonhydrolyzable GTP analog, incorporated per VP*) in the membrane preparations of purified carp rods and cones in response to light stimulation $(0.0085 \%$ for rods (a, circle) and $0.25 \%$ for cones (b, circle)) in the presence (filled symbols) and absence (open symbols) of ATP (1 mM) as determined by biochemical experiments [20]. Corresponding simulation results (dotted lines (-ATP) and dashed lines (+ATP) in a and b were superimposed onto the experimental results. B, Light-induced GTPYS binding as a function of flash intensity in rods (a, circle, after $40 \mathrm{sec}$ stimulation) and cones (b, circle, after $20 \mathrm{sec}$ stimulation) in the presence (filled symbols) and absence (open symbols) of ATP ( $0.1 \mathrm{mM})$ normalized to maximum values. Simulated results for rods ( $a$, triangle) and cones (b, triangle) reproduced under corresponding experimental conditions in the presence (filled symbols) and absence (open symbols) of ATP are also shown in the figure. Values for the rate constants for VP phosphorylation, kRK1 - kRK6 and kiRK1 - kiRK6, were set to 0 for simulation of experimental results obtained without ATP. 

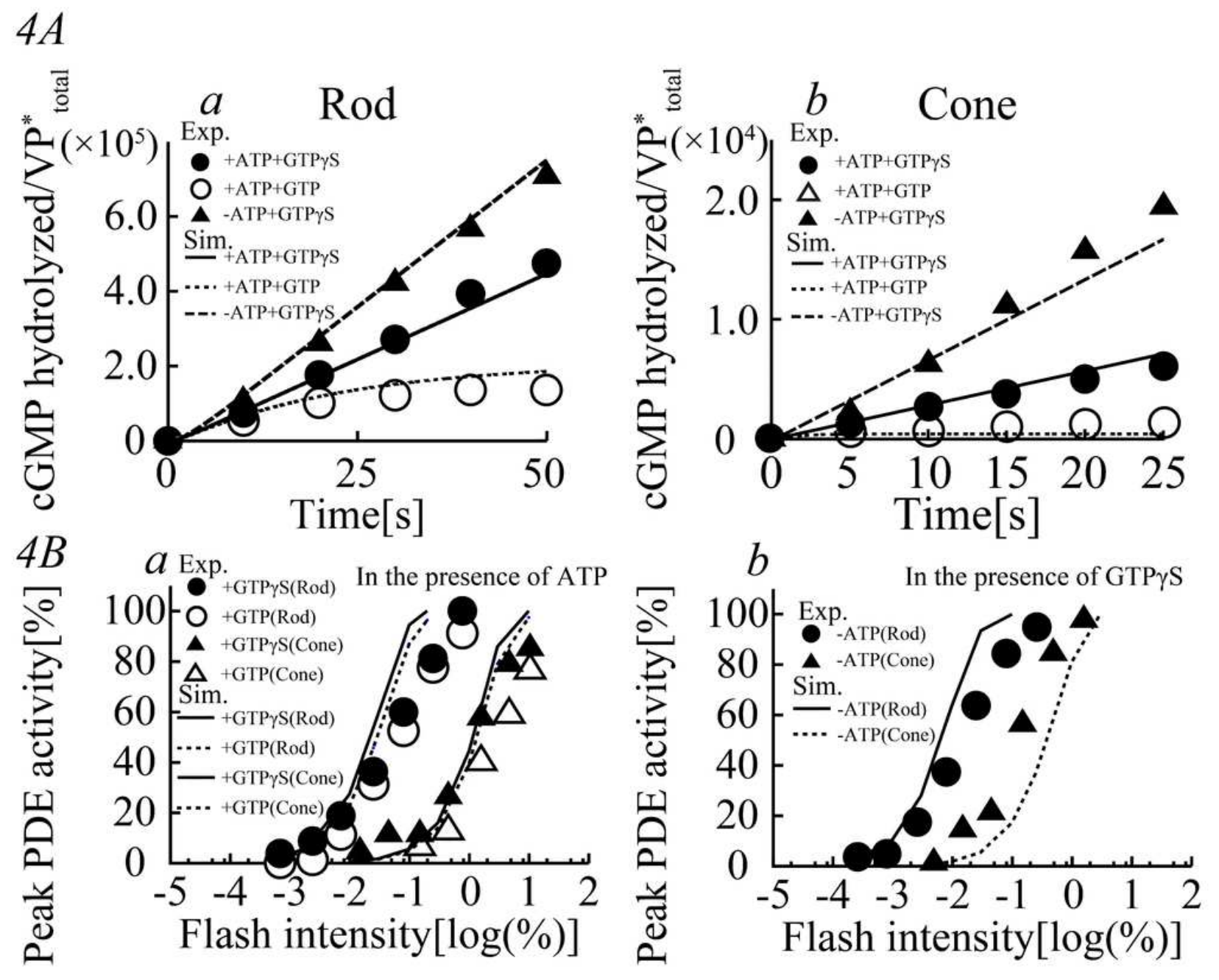

Figure 4

PDE-mediated cGMP hydrolysis in rods and cones A, The time courses for PDE activities (the number of cGMP molecules hydrolyzed per VP*) in the membrane of carp rods and cones in response to light stimulations (a, $0.024 \%$ for rods (open circle); $b, 0.46 \%$ for cones (open circle)) in the presence of ATP $(0.25 \mathrm{mM}), \mathrm{cGMP}(2500 \mu \mathrm{M})$ and either GTP (open closed circle) or GTPYS (filled triangle) determined by biochemical experiments [25] [19]). Corresponding simulation results (solid lines (with GTPYS) and dotted lines (with GTP) in a and b) were superimposed onto the experimental data. The continuous presence of saturating levels of cGMP was assumed for the simulation study, and thus $\beta$ dark was set to 0 . B, Lightinduced peak PDE activity as a function of flash intensity in rods (circle) and cones (triangle) either in the presence of ATP $(a, 0.25 \mathrm{mM})$ or without ATP (b) and either GTP (open symbols) or GTPYS (filled symbols) are normalized to maximum values. Simulated PDE activity of the rods and cones reproduced under the corresponding experimental conditions (with either GTP (dotted lines) or GTPYS (solid lines)) were superimposed onto the experimental results. The value for the rate constant, kdecay, for cGMP hydrolysis was set to 0 for the simulation of the experimental results obtained with GTPYS. 

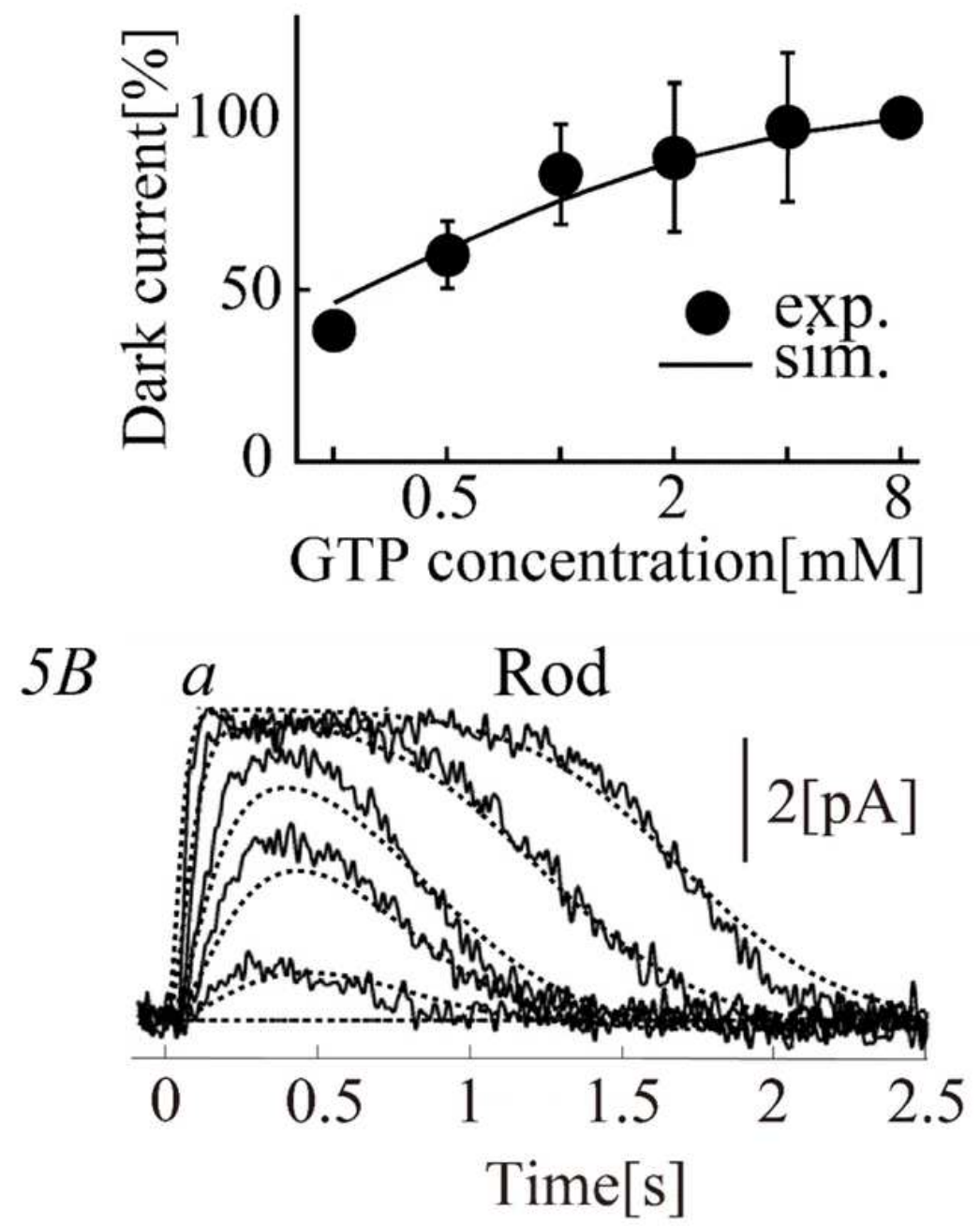

$b$ Cone

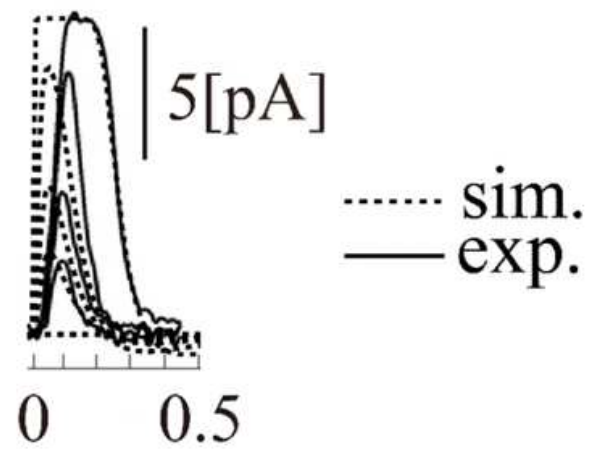

Time[s]

Figure 5

ICNG in rods and cones A, [GTP]i-dependent ICNG recorded from a truncated outer segment of frog rods (circle), normalized to ICNG at $8 \mathrm{mM}$ [GTP]i [14]. Corresponding simulation results at steady-state were superimposed onto the experimental data (solid line). B, Time courses of light-induced ICNG as a function of flash intensity recorded from the outer segments of the carp rods (a, light stimuli; 5.1E-6, 1.6E-5, 5.1E-5, 1.6E-4, and 5.1E-4\%) and cones (b, light stimuli; $1.6 \mathrm{E}-3,5.2 \mathrm{E}-3,1.6 \mathrm{E}-2$, and $0.16 \%$ ) as solid lines [14]. Simulated ICNG for rods (a) and cones (b) reproduced under the corresponding experimental conditions was superimposed onto the experimental results (dotted lines in $a$ and $b$ ).

\section{Supplementary Files}

This is a list of supplementary files associated with this preprint. Click to download.

- SciRepSuppV2.pdf 